\title{
Heparan Sulfate Proteoglycan Synthesis Is Dysregulated in Human Osteoarthritic Cartilage
}

\author{
Anastasios Chanalaris, ${ }^{*}$ Hannah Clarke, ${ }^{\dagger}$ Scott E. Guimond, ${ }^{\dagger}$ Tonia L. Vincent, ${ }^{*}$ Jeremy E. Turnbull, ${ }^{\dagger}$ and Linda Troeberg ${ }^{*}$
}

From the Arthritis Research UK Centre for Osteoarthritis Pathogenesis, * Kennedy Institute of Rheumatology, University of Oxford, Oxford; the Institute of Integrative Biology, ${ }^{\dagger}$ University of Liverpool, Liverpool, United Kingdom; and Norwich Medical School, ${ }^{\ddagger}$ University of East Anglia, Norwich, United Kingdom

Accepted for publication November 13, 2018.

Address correspondence to Linda Troeberg, Ph.D., Norwich Medical School, University of East Anglia, Bob Champion Research and Education Building, James Watson Road, Norwich, NR4 7UQ, United Kingdom. E-mail: 1.troeberg@uea.ac.uk.

\begin{abstract}
Osteoarthritis $(\mathrm{OA})$ is a common degenerative joint disease, characterized by cartilage loss and subchondral bone remodeling in response to abnormal mechanical load. Heparan sulfate (HS) proteoglycans bind to many proteins that regulate cartilage homeostasis, including growth factors, morphogens, proteases, and their inhibitors, and modulate their localization, retention, and biological activity. Changes in HS expression and structure may thus have important consequences for joint health. We analyzed normal and osteoarthritic human knee cartilage, and found HS biosynthesis was markedly disrupted in $0 \mathrm{~A}$, with $45 \%$ of the 38 genes analyzed differentially regulated in diseased cartilage. The expression of several HS core proteins, biosynthesis, and modification enzymes was increased in OA cartilage, whereas the expression of the HS proteoglycans syndecan 4 and betaglycan was reduced. The structure of HS was also altered, with increased levels of 6-0-sulfation in osteoarthritic samples, which correlated with increased expression of HS6ST1, a 6-0-sulfotransferase, and GLCE, an epimerase that promotes 6-0-sulfation. siRNA silencing of HS6ST1 expression in primary $0 \mathrm{~A}$ chondrocytes inhibited extracellular signal-regulated kinase phosphorylation in response to fibroblast growth factor 2, showing that changes in 6-0-sulfation impact a key cartilage signaling pathway. Given the broad range of homeostatic and repair pathways that HS regulates, these changes in proteoglycan expression and HS structure are likely to have significant effects on joint health and progression of OA. (Am J Pathol 2019, 189: 632-647; https://doi.org/ 10.1016/j.ajpath.2018.11.011)
\end{abstract}

Osteoarthritis (OA) is a common degenerative joint disease in which articular cartilage loss and subchondral bone remodeling cause pain and impair movement of affected joints. There are currently no disease-modifying therapies available, and patients are largely treated with analgesia or joint replacement surgery. There is thus a substantial need to develop effective therapeutics that can slow or halt the progression of joint damage.

The primary risk factors for development of OA are aging and injury, which alter the mechanical environment of the joint and stimulate chondrocytes to produce catabolic proteinases that degrade the cartilage extracellular matrix. The pericellular matrix immediately adjacent to chondrocytes is thought to play a central role in transducing mechanical stimuli to biochemical signals within the cells. ${ }^{1}$ For example, loading of cartilage causes release of fibroblast growth factor 2 (FGF2) from the pericellular matrix, and activates downstream extracellular signal-regulated kinase (ERK) signaling pathways in chondrocytes. ${ }^{2,3}$ FGF2 is localized in the pericellular matrix through its interaction with the heparan sulfate (HS) chains of perlecan, ${ }^{2}$ the major HS proteoglycan in cartilage. Pericellular matrix and cell surface HS also binds to many other bioactive proteins, including growth factors [eg, connective tissue growth

Supported by Arthritis Research UK grants 20887, 20205, and 21621 (all to A.C., T.L.V., and L.T.); and the National Institute for Health Research Oxford Biomedical Research Centre (A.C., T.L.V., and L.T.).

Disclosures: None declared.

The views expressed are those of the author(s) and not necessarily those of the National Health Service, the National Institute for Health Research, or the Department of Health. 
factor and transforming growth factor- $\beta$ (TGF- $\beta$ )], morphogens [eg, bone morphogenetic protein (BMP)-4 and BMP-7], proteinases (eg, matrix metalloproteinase 13 and adamalysin with thrombospondin motifs 4 and 5), and proteinase inhibitors (eg, tissue inhibitor of metalloproteinases-3), and regulates their retention, localization, and biological activity. ${ }^{4,5}$ HS is thus a critical regulator of chondrocyte homeostasis and cartilage health. Previous studies have shown increased expression of some of the HS core proteins in OA, including perlecan, ${ }^{6-9}$ syndecan $1,{ }^{9-11}$ and syndecan $4,{ }^{11-13}$ but nothing is yet known about how the structure of HS itself or its interactions with ligands change in OA.

HS interacts with proteins through electrostatic interactions between its negatively charged sulfate groups and positively charged lysine and arginine residues on the protein ligand. The number and arrangement of sulfate groups along the HS glycosaminoglycan chain determines the affinity of HS for its protein ligands and its ability to modulate their biological activity. ${ }^{14-16}$ This sulfation pattern is generated by the template-independent action of a series of sulfotransferases during HS synthesis in the Golgi apparatus. ${ }^{17}$ The exostosin (EXT) family of glycosyltransferases initiate HS synthesis by adding sequential glucuronic acid (GlcA) and $\mathrm{N}$-acetyl glucosamine (GlcNAc) residues to a linker tetrasaccharide attached to the core protein. GlcA can subsequently be epimerized to iduronic acid (IdoA) by glucuronyl C5-epimerase (GLCE). The growing HS chain can then be N-sulfated on GlcNAc residues [by the Ndeacetylase/N-sulfotransferases (NDSTs)], and/or Osulfated on GlcNAc [by the 6-O-sulfotransferases (HS6STs) and 3-O-sulfotransferases (HS3STs)] and GlcA/IdoA [by the 2-O-sulfotransferase (HS2ST1)]. ${ }^{17} \mathrm{HS}$ can also be edited in the extracellular environment by sulfatase 1 (SULF1) and sulfatase 2 (SULF2) that remove 6-O-sulfate groups, or degraded by the heparanases heparanase 1 (HSPE1) and heparanase 2 (HSPE2). These reactions do not always occur to completion, generating a highly variable sulfation pattern that gives HS a structural complexity several orders of magnitude larger than the proteome. Temporal and spatial changes in HS sulfation occur during development ${ }^{18-21}$ and in diseases, such as Alzheimer disease, ${ }^{22,23}$ cancer, ${ }^{24}$ diabetes, ${ }^{25}$ and fibrosis, ${ }^{26}$ and are thought to enable HS to coordinately regulate the retention and activity of multiple bioactive ligands, and thus to powerfully regulate tissue homeostasis.

Herein, we isolated RNA from knee cartilage of normal and osteoarthritic donors, and profiled expression of the 13 core proteins and 25 biosynthesis and modifying enzymes that govern HS structure and sulfation. Notably, disaccharide composition was examined by isolating HS from normal and OA cartilage. HS enzyme expression and glycan structure were found to be markedly dysregulated in OA cartilage, and increased 6-O-sulfation of HS was identified
Table 1 Primer/Probe Sets Used on Custom-Designed TaqMan Low-Density Arrays

\begin{tabular}{|c|c|}
\hline Gene name & Primer/probe set \\
\hline \multicolumn{2}{|c|}{ Chain elongation } \\
\hline EXT1 & Hs00609162_m1 \\
\hline EXT2 & Hs00181158_m1 \\
\hline EXTL1 & Hs00184929_m1 \\
\hline EXTL2 & Hs01018237_m1 \\
\hline EXTL3 & Hs00918601_m1 \\
\hline \multicolumn{2}{|c|}{$\mathrm{N}$-deacetylase/N-sulfotransferases } \\
\hline NDST1 & Hs00925442_m1 \\
\hline NDST2 & Hs00234335_m1 \\
\hline NDST3 & Hs01128584_m1 \\
\hline NDST4 & Hs00224024_m1 \\
\hline \multicolumn{2}{|c|}{ Glucuronyl C5-epimerase } \\
\hline GLCE & Hs00392011_m1 \\
\hline \multicolumn{2}{|c|}{ 2-0-sulfotransferase } \\
\hline HS2ST1 & Hs00202138_m1 \\
\hline \multicolumn{2}{|c|}{ 3-0-sulfotransferases } \\
\hline HS3ST1 & Hs00245421_s1 \\
\hline HS3ST2 & Hs00428644_m1 \\
\hline HS3ST3A1 & Hs00925624_s1 \\
\hline HS3ST3B1 & Hs00797512_s1 \\
\hline HS3ST4 & Hs00901124_s1 \\
\hline HS3ST5 & Hs00999394_m1 \\
\hline HS3ST6 & Hs03007244_m1 \\
\hline \multicolumn{2}{|c|}{ 6-0-sulfotransferases } \\
\hline HS6ST1 & Hs00757137_m1 \\
\hline HS6ST2 & Hs02925656_m1 \\
\hline HS6ST3 & Hs00542178_m1 \\
\hline \multicolumn{2}{|c|}{ Editing enzymes } \\
\hline$H S P E$ & Hs00935036_m1 \\
\hline HSPE2 & Hs00222435_m1 \\
\hline SULF1 & Hs00290918_m1 \\
\hline SULF2 & Hs01016476_m1 \\
\hline \multicolumn{2}{|l|}{ Core proteins } \\
\hline$A G R N$ & Hs00394748_m1 \\
\hline$G P C 1$ & Hs00892476_m1 \\
\hline GPC2 & Hs00415099_m1 \\
\hline GPC3 & Hs01018936_m1 \\
\hline GPC4 & Hs00155059_m1 \\
\hline GPC5 & Hs00270114_m1 \\
\hline GPC6 & Hs00170677_m1 \\
\hline HSPG2 & Hs00194179_m1 \\
\hline$S D C 1$ & Hs00896423_m1 \\
\hline SDC2 & Hs00299807_m1 \\
\hline SDC3 & Hs01568665_m1 \\
\hline SDC4 & Hs00161617_m1 \\
\hline TGFBR3 & Hs01114253_m1 \\
\hline \multicolumn{2}{|c|}{ Normalizing genes } \\
\hline $18 S$ & Hs99999901_s1 \\
\hline RPLPO & Hs99999902_m1 \\
\hline GAPDH & Hs99999905_m1 \\
\hline
\end{tabular}

AGRN, agrin; EXT, exostosin; EXTL, exostosin-like; GAPDH, glyceraldehyde-3-phosphate dehydrogenase; GPC, glypican; HSPE, heparanase; HSPG, HS proteoglycan 2; SDC, syndecan; RPLP0, ribosomal protein lateral stalk subunit PO; SULF, sulfatase; TGFBR3, transforming growth factor- $\beta$ receptor III. 

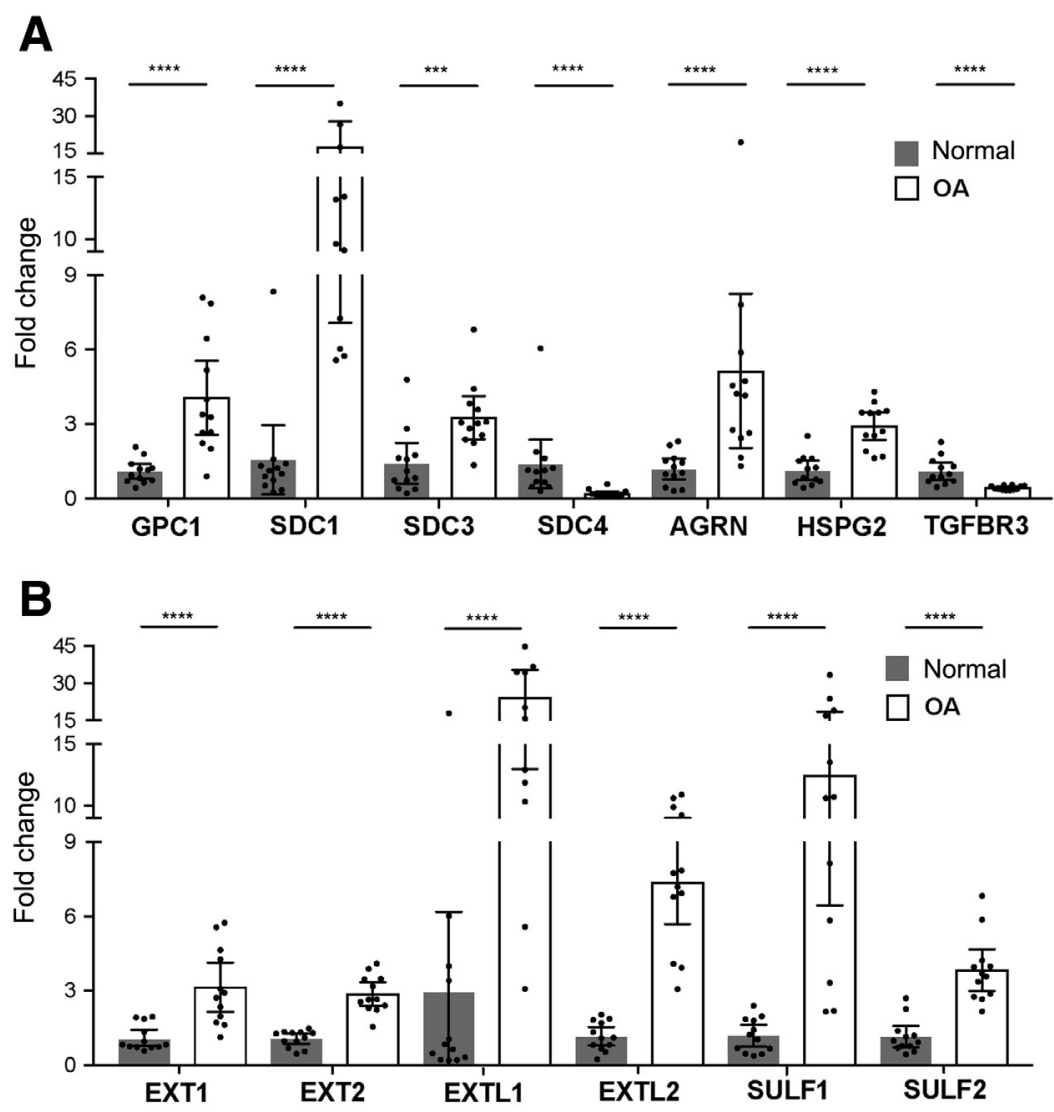

Figure 1 Changes in heparan sulfate (HS)-related gene expression in human osteoarthritic (OA) cartilage. Expression of HS-associated genes in normal and $\mathrm{OA}$ cartilage was analyzed using TaqMan low-density arrays. Fold changes in expression were determined by the $\Delta \Delta \mathrm{Ct}$ method against the average of the normal samples. A: Fold changes in expression of HS core protein genes. B: Fold changes in expression of HS chain elongation and sulfatase genes. C: Fold changes in expression of sulfotransferase genes. Error bars represent $95 \%$ CIs of the mean. $n=12$ per group $(\mathbf{A}-\mathbf{C}) .{ }^{* *} \mathrm{q}<0.01$, ${ }^{* * *} \mathrm{q}<0.001$, and ${ }^{* * * *} \mathrm{q}<0.0001$ (Benjamini, Krieger, and Yekutieli-corrected multiple $t$-test). AGRN, agrin; EXT, exostosin; EXLT, exostosin-like; GLCE, glucuronyl C5epimerase; GPC, glypican; HS3ST, 3-0-sulfotransferase; HS6ST, 6-0-sulfotransferase; HSPG, heparan sulfate proteoglycan; NDST, N-deacetylase/N-sulfotransferase; SDC, syndecan; SULF, sulfatase; TGFBR3, transforming growth

C factor- $\beta$ receptor III.

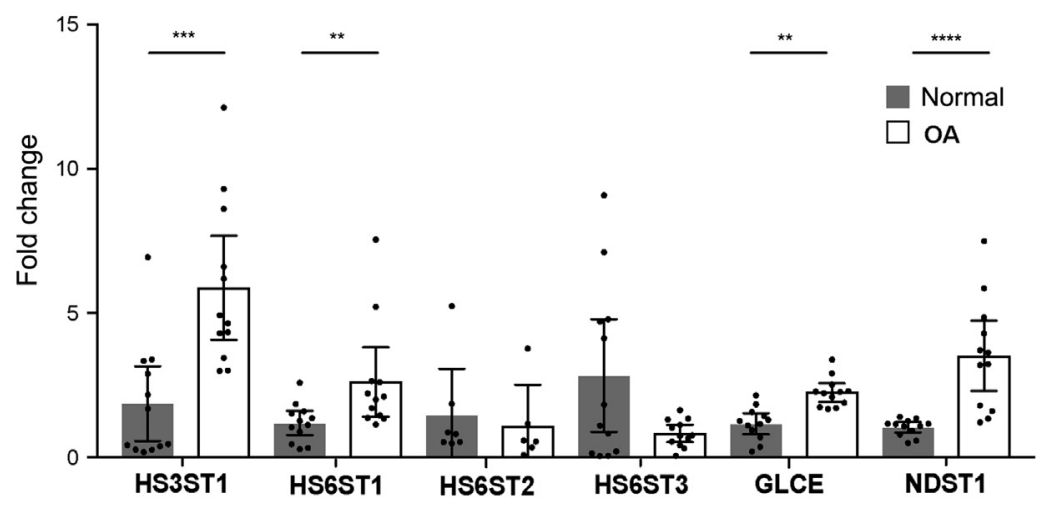

as the primary structural change associated with impaired cartilage homeostasis.

\section{Materials and Methods}

\section{Human Cartilage Samples}

Human articular cartilage was obtained in full compliance with national and institutional ethical requirements, the UK Human Tissue Act, and the Declaration of Helsinki.

Normal human knee cartilage specimens used for RNA and glycosaminoglycan analyses were obtained from Articular Engineering LLC (Northbrook, IL) from donors (six females of 44 to 68 years of age, six males of 32 to 75 years of age) with clinically healthy, normal cartilage and no history of OA, following informed donor consent and approval by local ethics committees and the University of Oxford Research Ethics Committee (R45926/RE001). Cartilage was aseptically harvested within 48 hours of death, flash frozen in liquid nitrogen, stored at $-80^{\circ} \mathrm{C}$, and shipped in dry ice.

Normal human knee cartilage specimens used for immunohistochemistry were obtained from Stanmore Biobank (Royal National Orthopedic Hospital, Stanmore, UK) from donors undergoing amputation for low limb malignancies with no involvement of the cartilage, following informed donor consent and approval by the Royal Veterinary College Ethics and Welfare Committee (institutional approval unique reference number 2012 0048H). Cartilage was aseptically 


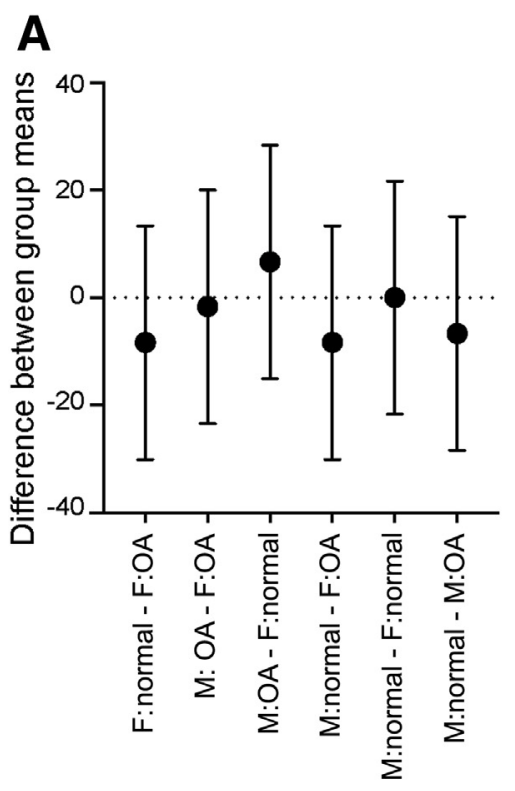

B

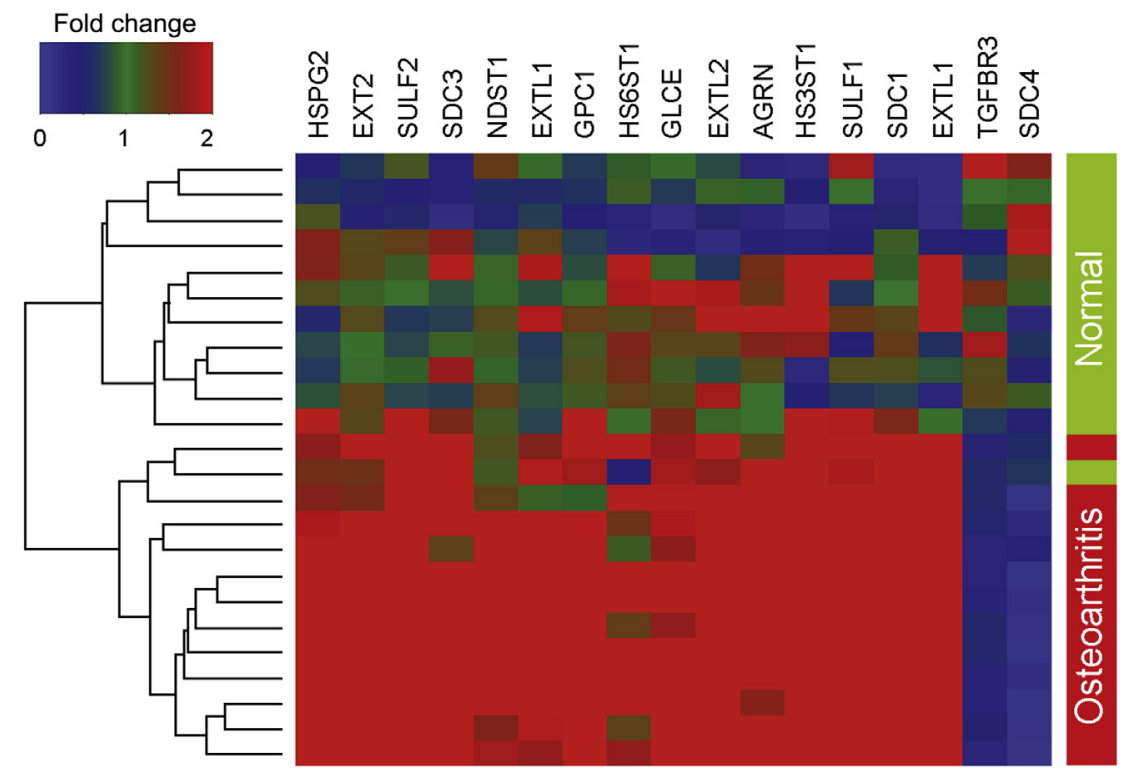

Figure 2 Expression of heparan sulfate (HS)-associated genes is altered in osteoarthritic (OA) cartilage. A: Forest plot showing there is no significant difference between the ages and sexes of normal and OA cartilage donors used for gene expression analysis. Bars show $95 \%$ CIs of the mean, dotted line shows $Y=0$. B: Expression of HS-associated genes in normal and OA cartilage was analyzed using TaqMan low-density arrays. Fold change in expression was determined by the $\Delta \Delta \mathrm{Ct}$ method against the average of the normal samples. The heatmap shows only those HS-associated genes whose expression was significantly different in the two groups (Benjamini, Krieger, and Yekutieli-corrected multiple $t$-test; $q<0.05$ ), with increased expression in $0 \mathrm{~A}$ shown in red, reduced expression shown in blue, and no change indicated in green. Cluster analysis based on the expression levels of significantly regulated genes separated the samples into two clusters, with the first cluster containing all of the OA samples and one of the normal samples, and the second cluster containing the remaining normal samples. $n=12$ for each group (A and B). F, female; M, male; AGRN, agrin; EXT, exostosin; EXLT, exostosin-like; GLCE, glucuronyl C5-epimerase; GPC, glypican; HS3ST, 3-0-sulfotransferase; HS6ST, 6-0-sulfotransferase; HSPG, heparan sulfate proteoglycan; NDST, N-deacetylase/N-sulfotransferase; SDC, syndecan; SULF, sulfatase; TGFBR3, transforming growth factor- $\beta$ receptor III.

harvested from all surfaces of the joint within 4 hours of surgery and processed for downstream analyses.

Human OA cartilage samples used for immunohistochemistry, RNA, and glycosaminoglycan analyses were obtained from the Oxford Musculoskeletal Biobank from donors (six females of 58 to 83 years of age, six males of 52 to 84 years of age) undergoing total knee replacement surgery for advanced OA, following informed consent and approval by the Oxford Research Ethics Committee C (09/ H0606/11). Cartilage was aseptically harvested from all surfaces of the joint within 4 hours of surgery and processed for downstream analyses.

\section{RNA Isolation}

Cartilage $(300 \mathrm{mg}$ ) from 12 normal and 12 OA donors was ground under liquid nitrogen with a pestle and mortar. The ground cartilage powder was added to $1.5 \mathrm{~mL}$ of RLT lysis buffer supplemented with $10 \mu \mathrm{L} / \mathrm{mL} \quad \beta$-mercaptoethanol (Qiagen, Manchester, UK), digested with proteinase K (6 to 10 $\mathrm{mAU} / 100 \mathrm{mg}$ of cartilage, 10 minutes at $55^{\circ} \mathrm{C}$; Qiagen, Manchester, UK), and transferred to an RNeasy micro column (Qiagen, Manchester, UK). RNA was extracted according to the manufacturer's protocol with an intermediate DNA digestion step. The eluted RNA was quantified on a Nanodrop spectrophotometer and examined on a Bioanalyser (Agilent,
Stockport, UK). RNA from healthy and OA cartilage had comparable RNA integrity numbers (6 to 7.7) and RNA yields.

\section{RT-PCR and Quantitative PCR}

Total RNA (250 ng) was reverse transcribed to cDNA using a reverse transcription kit (ThermoFisher Scientific, Loughborough, UK), and cDNA was amplified on a TaqMan lowdensity array (Table 1). Fold changes against the control samples were calculated using the $\Delta \Delta \mathrm{Ct}$ method. For calculating the $\Delta \mathrm{Ct}$, the average of the three normalizing genes (18S, RLPLO, and GAPDH) was used.

\section{Extraction of HS Proteoglycans and Disaccharide Analysis}

Approximately $300 \mathrm{mg}$ of frozen cartilage explants was ground in a pestle and mortar under liquid nitrogen, and the powder was placed in $1 \mathrm{~mL}$ of Trizol (ThermoFisher Scientific). After phase separation with the addition of chloroform, the aqueous phase was added to equilibrated diethylaminoethyl-Sephacel beads and washed with phosphate-buffered saline (PBS) and $0.25 \mathrm{~mol} / \mathrm{L} \mathrm{NaCl}$ before elution with $2 \mathrm{~mol} / \mathrm{L} \mathrm{NaCl}$. The eluate was desalted on PD10 columns and freeze dried. The samples were then treated with DNase (Qiagen, Manchester, UK) and RNase, 
A
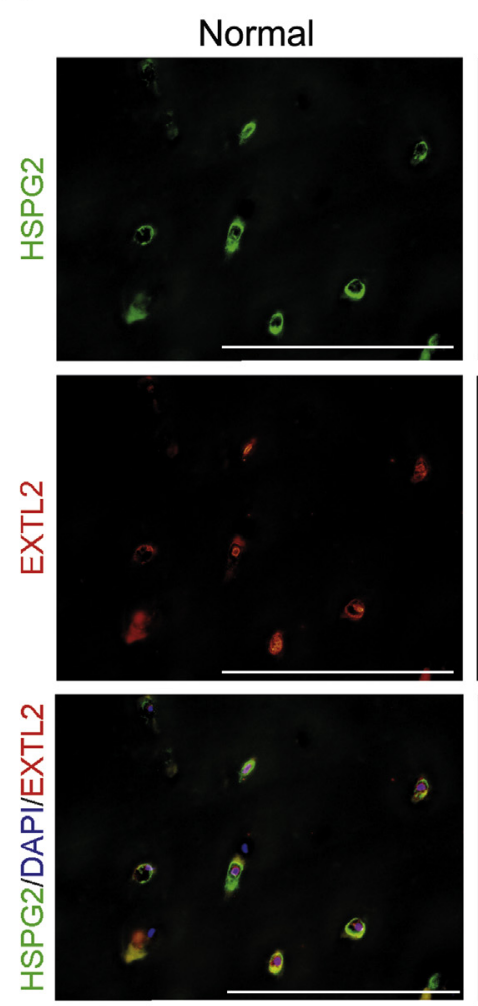

C
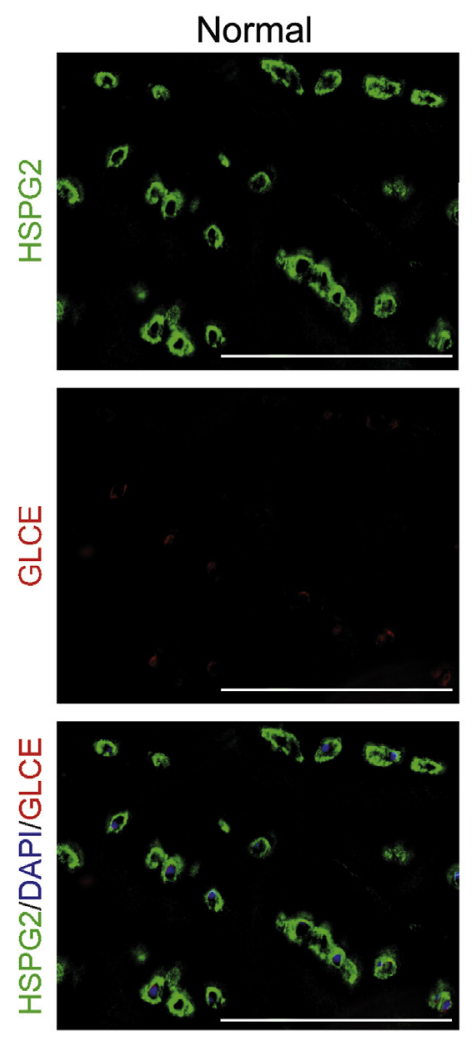
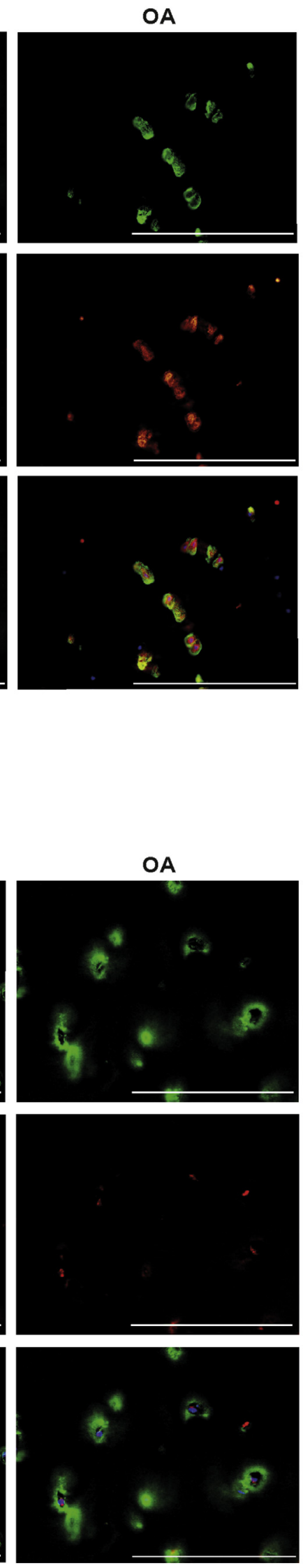

B

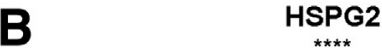

HSPG2
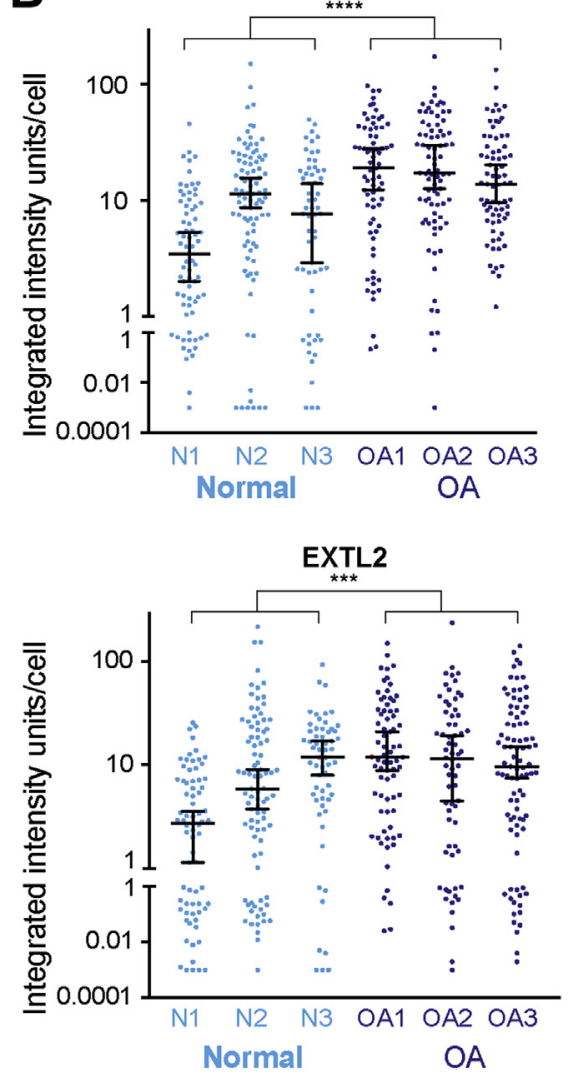

D
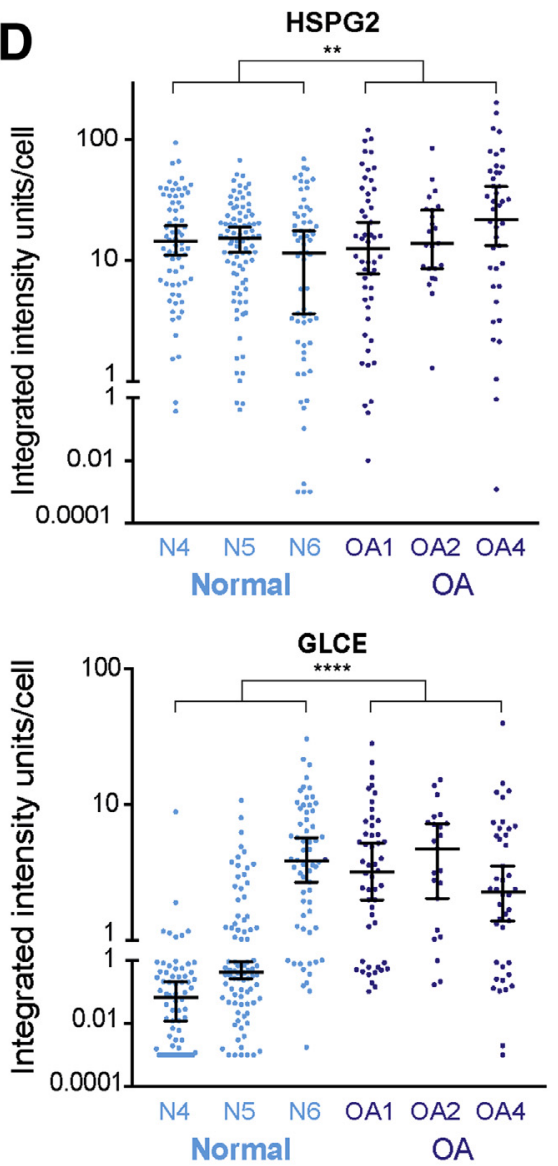
before incubation with chondroitinase ABC (Sigma Aldrich, Gillingham, UK), neuraminidase, keratanase, and pronase. After the digestions, the samples were once more purified on diethylaminoethyl beads and freeze dried. The rest of the procedure was as described before. ${ }^{27}$ Briefly, samples were sequentially digested with heparanase I, II, and III (IBEX Pharmaceuticals, Montreal, Canada), purified on $\mathrm{C} 18$ and graphite spin columns (ThermoFisher Scientific), and labeled with BIODIPY hydrazine (ThermoFisher Scientific), as previously described. ${ }^{28}$ Finally, samples underwent ethanol precipitation to remove any copurified impurities. Samples were then analyzed by SAX high-performance liquid chromatography using a Propac PA-1 strong anion-exchange column $(4.6 \times 250 \mathrm{~nm}$; Dionex, Loughborough, UK). Samples were eluted with a linear gradient of 0 to $-1 \mathrm{~mol} / \mathrm{L}$ sodium chloride and isocratic sodium hydroxide $(150 \mathrm{mmol} / \mathrm{L})$ over 30 minutes at $1 \mathrm{~mL} /$ minute on a Shimadzu high-performance liquid chromatography system (Shimadzu, Milton Keynes, UK). An inline fluorimeter was used to detect eluted peaks (excitation $\lambda=488 \mathrm{~nm}$, emission $\lambda=520 \mathrm{~nm}$ ). Previously calculated correction factors were applied to quantitate the observed disaccharides. ${ }^{27}$

\section{Immunofluorescence Staining Analysis}

On receipt, cartilage explants were embedded in OCT (Thermofisher Scientific) and frozen in isopentane for storage at $-80^{\circ} \mathrm{C}$ until sectioning. Samples were mounted on a cryostat (CM1860UV; Leica, Milton Keynes, UK) and sections ( $5 \mu \mathrm{m}$ thick) were cut and mounted on Superfrost Plus microscope slides (ThermoFisher Scientific) and stored at $-80^{\circ} \mathrm{C}$. Frozen sections were air dried for 1 hour at room temperature and fixed with neutral-buffered formalin for 5 minutes at room temperature before their incubation in icecold acetone for 10 minutes at $-20^{\circ} \mathrm{C}$, and incubated at $37^{\circ} \mathrm{C}$ for 30 minutes with $0.1 \mathrm{U}$ chondroitinase $\mathrm{ABC}$ (C3667; Sigma-Aldrich) in $1 \times$ chondroitinase buffer (40 $\mathrm{mmol} / \mathrm{L}$ tris-acetate, $\mathrm{pH} 8$ ). The digested sections were blocked in $1 \%$ goat serum and $5 \%$ bovine serum albumin in PBS for 1 hour at room temperature. After 15 minutes of permeabilization with $0.1 \%$ Triton X-100 in PBS, sections were washed in PBS and the primary antibodies or isotype negative control antibodies were applied diluted in block buffer overnight at $4{ }^{\circ} \mathrm{C}$. The following antibodies were used: rat antiperlecan [HS proteoglycan 2 (HSPG2); 1:1000; Millipore, Watford, UK], rabbit anti-GLCE (1:20; HPA04821; Atlas Antibodies, Bromma, Sweden), rabbit anti-exostosinlike (EXTL)2 (1:100; NBP2-16394; Novus Biologicals,
Abingdon, UK), mouse anti-NDST1 (3 $\mu \mathrm{g} / \mathrm{mL}$; ab55296; Abcam, Cambridge, UK), rabbit anti-glypican 1 (GPC1; 1:250; HPA303571; Sigma-Aldrich), and rabbit anti-HS6ST1 (1:250; ab106195; Abcam). After washing in PBS at room temperature, the appropriate secondary antibodies were added in block buffer, either in combination or in unison: goat anti-rat Alexa Fluor 488 (1:1000; ThermoFisher Scientific), goat anti-rabbit Alexa Fluor 568 (1:500; ThermoFisher Scientific), or goat anti-mouse Alexa Fluor 568 (1:500; ThermoFisher Scientific). The secondary antibodies were washed off with PBS after 3 hours at room temperature. Nuclei were stained with DAPI (1:200 in PBS; ThermoFisher Scientific) for 1 hour before a final wash in PBS and mounting in Prolong Diamond antifade (ThermoFisher Scientific).

Sections were viewed under an Olympus BX51 fluorescent microscope (Olympus, Southend-on-Sea, UK). For each protein, the optimal duration of exposure was determined as that giving a signal in positively stained sections and no detectable staining in negatively stained sections, and avoiding overexposure and signal saturation. The exposures used were as follows: HSPG2, 200 to 250 milliseconds; EXTL2, 125 milliseconds; GLCE, 200 milliseconds; GPC1, 125 milliseconds; NDST1, 280 to 300 milliseconds; and HS6ST1, 275 to 300 milliseconds. Images were obtained from at least six random regions of the cartilage under a $40 \times$ objective lens and then analyzed on ImageJ software version $1.52 \mathrm{~h}$ (NIH, Bethesda, MD; http://imagej.nih.gov/ij), as described previously. ${ }^{29,30}$ For representative images, the intensity of the red and green signal was adjusted for illustrative purposes. For quantification, raw unaltered images were analyzed.

\section{Analysis of ERK Phosphorylation in Chondrocytes}

Primary OA chondrocytes were isolated from freshly harvested human OA cartilage by chopping the tissue finely and incubating in Dulbecco's modified Eagle's medium with 10\% fetal calf serum (ThermoFisher Scientific) and $1.5 \mathrm{mg} / \mathrm{mL}$ type II collagenase (Roche Diagnostics, Burgess Hill, UK) (18 hours, $37^{\circ} \mathrm{C}$ ). The suspension was passed through a cell strainer, pelleted, and washed twice with medium. Isolated chondrocytes were cultured for 7 days until confluent ${ }^{31}$ and stored at $-80^{\circ} \mathrm{C}$ until required. For siRNA transfection, chondrocytes $\left(3 \times 10^{5}\right.$ per well) were transfected with 20 $\mathrm{nmol} / \mathrm{L}$ of either Silencer Select Negative control number 1 or Silencer Select HS6ST1 siRNA (s17978; ThermoFisher Scientific, UK) using Lipofectamine 2000 (ThermoFisher Scientific, UK) in serum-free Opti-MEM I (ThermoFisher Scientific). ${ }^{31}$ After 4 hours, the medium was replaced with

\footnotetext{
Figure 3 Expression of HS proteoglycan 2 (HSPG2), exostosin-like (EXTL)2, and glucuronyl C5-epimerase (GLCE) is increased in human osteoarthritic (OA) cartilage. A: Representative images showing expression of HSPG2 and EXLT2 in human normal and OA cartilage. B: Expression of HSPG2 and EXLT2 was semiquantitatively evaluated in three normal donors (N1, N2, and N3) and three $0 A$ donors $(0 A 1,0 A 2$, and $0 A 3)$, with each dot representing integrated fluorescence intensity per single cell, calculated from at least six random fields of view on three sections per donor. For each donor, the median fluorescence intensity with $95 \%$ CIs of the mean are shown. C: Representative images showing expression of HSPG2 and GLCE in human normal and OA cartilage. D: Expression of HSPG2 and GLCE was semiquantitatively evaluated in three normal donors (N4, N5, and N6) and three 0A donors (0A1, 0A2, and 0A4), as described in B. ${ }^{* *} P<0.01,{ }^{* *} P<0.001$, and ${ }^{* * * *} P<0.0001$ (Kolmogorov-Smirnov test). Scale bars $=260 \mu \mathrm{m}(\mathbf{A}$ and $\mathbf{C})$.
} 
A
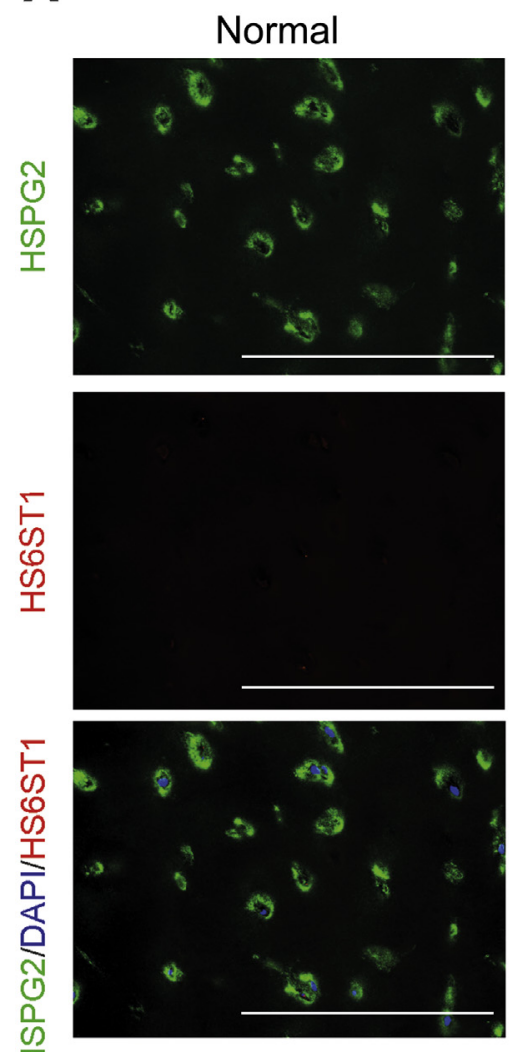

I

C
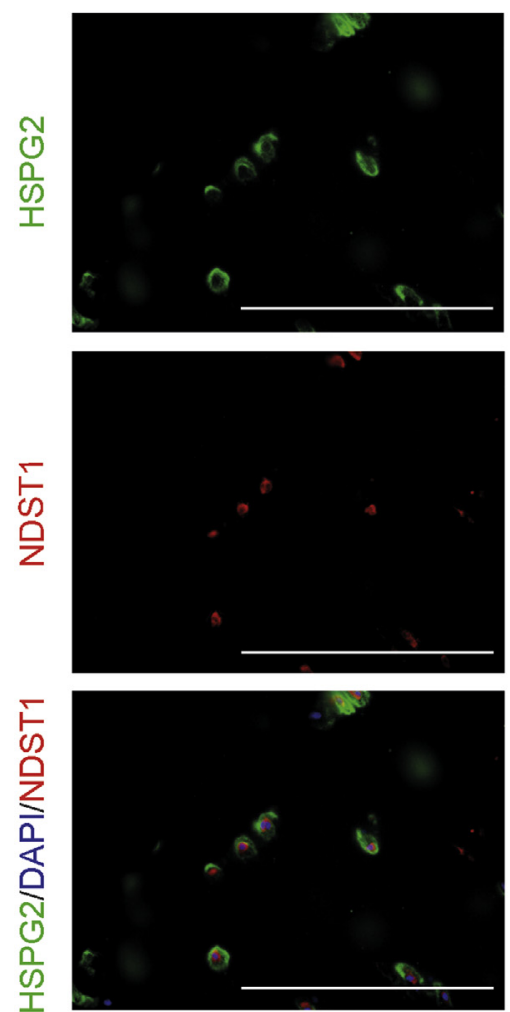

$O A$
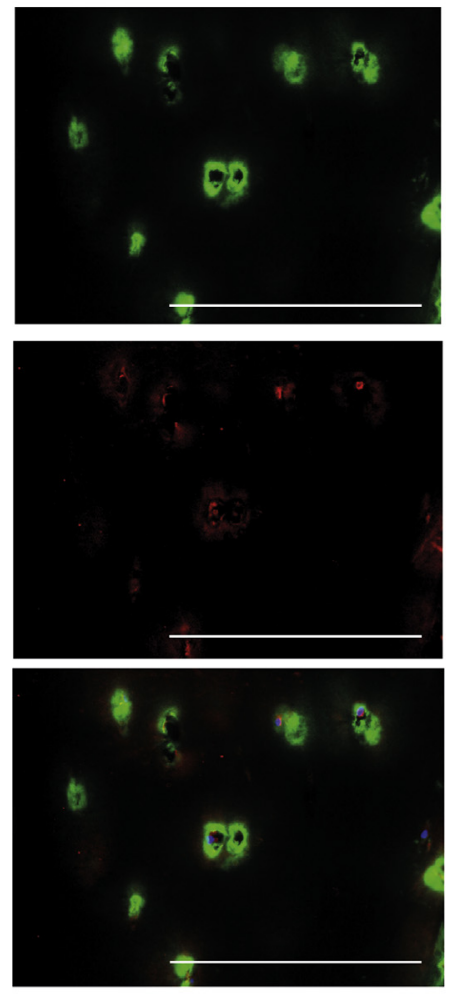

$O A$
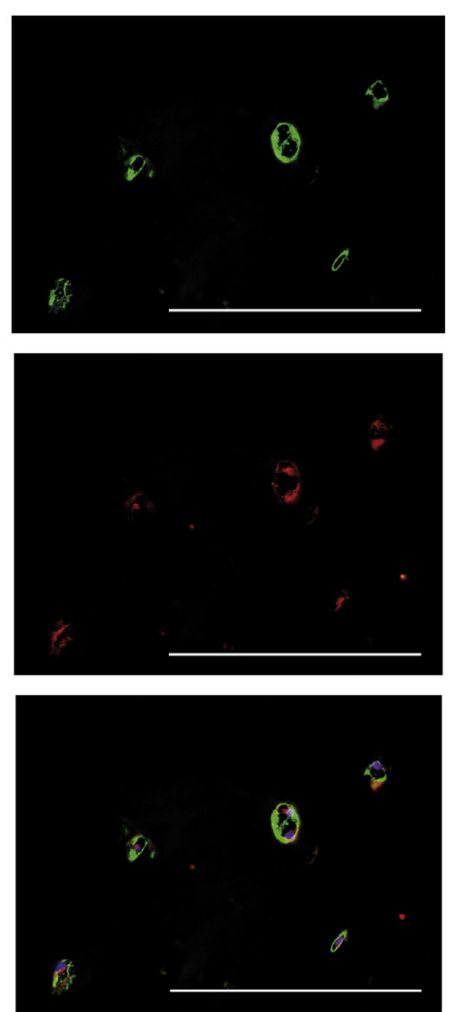

\section{B HSPG2}

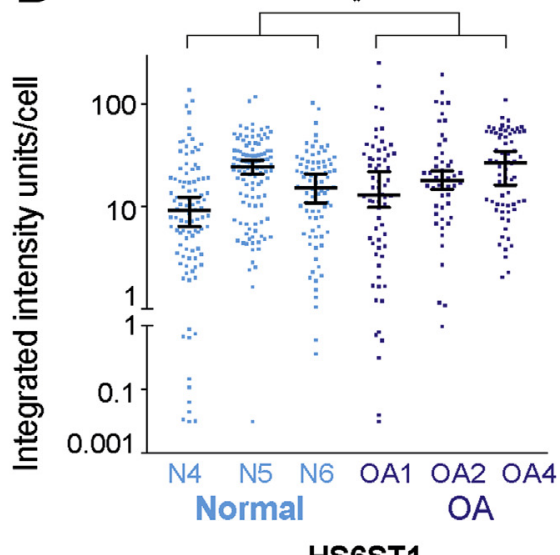

HS6ST1
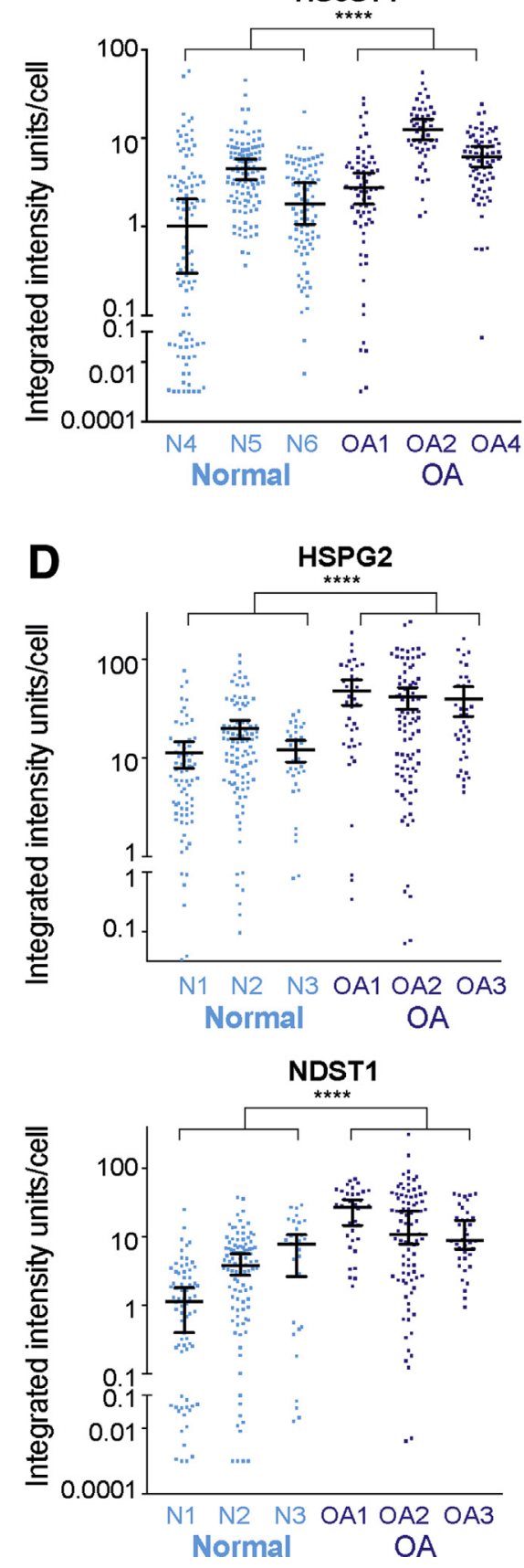
Dulbecco's modified Eagle's medium containing 10\% fetal calf serum and cell cultured for a further 72 hours.

To analyze ERK phosphorylation, siRNA-treated chondrocytes were cultured in serum-free Dulbecco's modified Eagle's medium for 24 hours and treated with recombinant human FGF2 (R\&D Systems, Minneapolis, MN) $(20 \mathrm{ng} / \mathrm{mL}$, 10 minutes). Cells were then washed twice with ice-cold PBS and lysed on ice in $150 \mu \mathrm{L}$ radioimmunoprecipitation assay buffer containing protease inhibitors (SIGMAFAST; SigmaAldrich) and phosphatase inhibitors (20 mmol/L NaF, 100 $\mathrm{mmol} / \mathrm{L} \mathrm{Na} \mathrm{VO}_{4}, 100 \mathrm{mmol} / \mathrm{L} \beta$-glycerophosphate, and 100 $\mathrm{mmol} / \mathrm{L}$ sodium pyrophosphate). Lysates were centrifuged ( $2500 \times g, 3$ minutes), and $20 \mu \mathrm{g}$ of protein was analyzed by immunoblotting using antibodies against phosphorylated ERK1/2 (1:2500; E7028; Sigma-Aldrich) and total ERK (1:5000; 4695S; Cell Signaling Technology, London, UK) in block buffer (5\% milk/PBS with Tween 20). After $3 \times 5$ minute washes with PBS with Tween 20, the membranes were incubated with an alkaline phosphatase-conjugated anti-rabbit secondary antibody (1:2000; Promega, UK) and developed using Western Blue stabilized substrate (Promega, Southampton, UK). Immunoblots were analyzed by densitometry using Phoretix 1D software version 11.5 (TotalLab, Newcastle-upon-Tyne, UK), and the levels of phosphorylated ERK were normalized to total ERK for each treatment, and expressed relative to the corresponding untreated sample (defined as 1).

Efficacy of siRNA silencing was confirmed by isolating RNA from radioimmunoprecipitation assay buffer lysates (20 to $50 \mu \mathrm{L}$, diluted to $350 \mu \mathrm{L}$ with RLT buffer containing $1 \%$ $\mathrm{v} / \mathrm{v} \beta$-mercaptoethanol) using RNeasy micro kits, according to the manufacturer's protocol (Qiagen, Manchester, UK). Isolated RNA was reverse transcribed, and expression of $18 S$, $G A P D H$, and $H S 6 S T I$ was quantified as described above.

\section{Statistical Analysis}

All statistical tests, except correlations and linear regression models, were performed in GraphPad Prism version 7.0d (GraphPad Software, La Jolla, CA). All other statistical tests were performed in R version 3.4.4 (The R Foundation, Vienna, Austria; https://www.r-project.org). All significant changes are annotated in the figures.

Power calculations were based on pilot data from four samples per group. According to the pilot experiments, the sample size required for a two-tailed $t$-test was calculated with an effect size of 1.5 (difference between means of 0.6 and SD of 0.4) and a type I error rate $\alpha$ at $5 \%$, to be 12 samples per group. Therefore, the groups were extended to the appropriate sizes.

Before performing any parametric test, groups were tested for normality using the D'Agostino and Pearson omnibus test. ${ }^{32}$

Two-way analysis of variance was performed, and the forest plots (Figure 1) were examined to evaluate whether there was significant variation in the age or sex of the donors.

All tests on the PCR results were performed on the $\Delta \Delta \mathrm{Ct}$ values because they were normally distributed. Multiple two-tailed $t$-tests were performed, comparing the expression of each gene between the two groups. The derived $P$ values were corrected for multiplicity using the two-stage step-up method of Benjamini, Krieger, and Yekutieli, with a falsediscovery rate cutoff at $5 \% .^{33}$

For HS disaccharide composition, site of sulfation, and extent of sulfation, two-way analysis of variance was performed, with a Benjamini, Krieger, and Yekutieli multiplicity correction.

Hierarchical clustering was performed in $\mathrm{R}$ using the package gplots $\beta$ and RColorBrewer. Canberra distance was used as the clustering metric.

For analysis of immunofluorescence images, the background-corrected integrated intensity values per cell were aggregated for the normal and OA groups ( $n=3$ to 6 per group), and the cumulative distributions of signal were analyzed using the Kolmogorov-Smirnov test because the values were not normally distributed.

\section{Results}

\section{Expression of HS Proteoglycan Core Proteins Is Disrupted in OA Cartilage}

RNA was isolated from normal and osteoarthritic tibial knee cartilage ( $n=12$ per group), and expression of 13 genes encoding HS proteoglycan core proteins was examined. Genes with a significant change in expression are shown in Figure 1.

Expression of agrin $(A G R N)$ and perlecan $(H S P G 2)$ was significantly increased in OA samples (multiple $t$-tests, Benjamini, Krieger, and Yekutieli-corrected $\mathrm{q}<0.05$ ), whereas expression of betaglycan (TGF- $\beta$ receptor III, $T G F B R 3$ ) was reduced.

Among the syndecan family, syndecan 2 (SDC2) was not differentially regulated between the groups, whereas expression of syndecan 4 (SDC4) was reduced in OA, and expression of syndecan $1(S D C 1)$ and syndecan $3(S D C 3)$ was

\footnotetext{
Figure 4 Expression of perlecan (heparan sulfate proteoglycan 2; HSPG2), 6-0-sulfotransferase 1 (HS6ST1), and N-deacetylase/N-sulfotransferase 1 (NDST1) is increased in human osteoarthritic (OA) cartilage. A: Representative images showing expression of HSPG2 and HS6ST1 in human normal and 0A cartilage. B: Expression of HSPG2 and HS6ST1 was semiquantitatively evaluated in three normal donors (N4, N5, and N6) and three 0A donors (0A1, 0A2, and $0 A 4$ ), with each dot representing integrated fluorescence intensity per cell, calculated from at least six random fields of view on three sections per donor. For each donor, the median fluorescence intensity with 95\% CIs of the mean are shown. C: Representative images showing expression of HSPG2 and NDST1 in human normal and OA cartilage. D: Expression of HSPG2 and NDST1 was semiquantitatively evaluated in three normal donors (N1, N2, and N3) and three 0A donors (0A1, 0A2, and OA3), as described in B. ${ }^{*} P<0.05,{ }^{* * *} P<0.0001$ (Kolmogorov-Smirnov test). Scale bars $=260 \mu \mathrm{m}(\mathbf{A}$ and $\mathbf{C})$.
} 
increased. $S D C l$ was the most strongly regulated of the HS core proteins, with mean expression increasing 17 -fold in OA.

Glypican core proteins showed fewer changes in expression in OA cartilage, with only GPCl showing significantly higher expression in OA samples. Expression of $G P C 4, G P C 5$, and GPC6 was not significantly regulated, and $G P C 2$ and $G P C 3$ were minimally expressed in both normal and OA cartilage.

\section{Expression of HS Biosynthesis and Modifying Enzymes Was Also Disrupted in OA Cartilage}

Using the same samples, expression of 25 genes encoding HS biosynthesis and modifying enzymes was analyzed (Figure 1), and 10 of these were found to be expressed at higher levels in OA cartilage.

Expression of EXT1 and EXT2, which extend the growing HS chain, was increased in OA, as was expression of the related EXTL1 and EXTL2 genes. EXTL1 was the most strongly regulated of all of the genes examined, showing a 24-fold increase in expression in OA. Expression of EXLT3 was not significantly regulated. Mean expression of epimerase $(G L C E)$, which epimerizes glucuronic acid to IdoA, was increased twofold in OA cartilage.

Mean expression of the $\mathrm{N}$-sulfotransferase NDST1 was threefold higher in OA cartilage. NDST2 was expressed at similar levels in both sample groups, and NDST3 and NDST4 were not appreciably expressed in either normal or OA cartilage.

Expression of the 2-O-sulfotransferase $H S 2 S T 1$ was not significantly altered in OA samples. Of the seven isoforms of 3-O-sulfotransferase, only HS3STI showed increased expression in OA cartilage, with expression of HS3ST3Al and $H S 3 S T 3 B 1$ not differentially regulated and four of the isoforms (HS3ST2, HS3ST3, HS3ST5, and HS3ST6) not detectably expressed in cartilage. Among the three 6-Osulfotransferase isoforms, expression of HS6ST1 was increased in OA cartilage, whereas HS6ST3 expression was similar in both sample groups and HS6ST2 was expressed at low levels.

Expression of both $S U L F 1$ and $S U L F 2$ was increased in OA cartilage, with $H S P E$ and HSPE2 not differentially expressed.

Cluster analysis of the samples, based on the levels of the significantly regulated genes, separated the samples into two clusters (Figure 2), with the first cluster containing all of the OA samples and one of the normal samples, and the second cluster containing the remaining normal samples. This indicates that levels of expression of the 17 significantly regulated genes are largely able to discriminate normal and osteoarthritic cartilage.

\section{Selected Immunohistochemical Analysis Validates Observed Changes in Expression}

To establish whether the observed gene regulation resulted in corresponding changes in protein expression, six proteins (HSPG2, EXTL2, GLCE, NDST1, HS6ST1, and GPC1) were selected to be analyzed by semiquantitative immunofluorescence staining on frozen sections of normal and OA cartilage. HSPG2 was selected as a marker of pericellular matrix localization, and the other five proteins were selected according to functional antibody availability and to have at least one from each category of HS core proteins and biosynthetic enzymes. The intensity of staining did not vary with cartilage depth for any of the antibodies examined.

As expected, HSPG2 exhibited a predominantly pericellular localization, ${ }^{34}$ with approximately $50 \%$ increased expression per cell in the four OA samples examined compared with the six normal cartilage samples.

EXTL2 had a predominantly perinuclear localization in both normal and OA cartilage (Figure 3A). In OA cartilage, expression of EXTL2 was increased 1.6-fold, with a decrease in the number of cells not expressing EXTL2 and a shift toward more cells expressing moderate amounts of the protein (Figure 3B).

GLCE was similarly localized in the perinuclear region of cells (Figure 3C), with 2.4-fold higher expression in OA and a shift toward more cells expressing modest amount of the enzyme (Figure 3D).

HS6ST1 intensity levels were approximately three times higher in OA (Figure 4B). The protein appeared to be expressed at low levels around the nucleus in normal cartilage and to show some pericellular staining in the OA samples (Figure 4A).

NDST1 was localized in the perinuclear region in normal cells, with more expression toward the periphery of the cell and overlapping with HSPG2 in the OA samples (Figure 4C). Intensity levels were approximately 3.6-fold higher in the OA samples (Figure 4D).

Staining for GPC1 was predominantly intracellular (Figure 5A), with 5.5-fold higher intensity in OA samples (Figure 5B).

\section{Increased 6-0-Sulfation of HS in OA Cartilage}

The amount and disaccharide composition of HS isolated from normal and OA cartilage was analyzed $(n=8$ per group) (Figure 6A).

There was no significant difference in the total amount of $\mathrm{HS}$ in the normal and OA cartilage samples $(5.7 \pm 2.3 \mu \mathrm{g} / 100$ $\mathrm{mg}$ in the normal samples, $3.02 \pm 0.73 \mu \mathrm{g} / 100 \mathrm{mg}$ of tissue in OA; $P>0.05$ by $t$-test) (Figure $6 \mathrm{~B}$ ), or in the ratio of sulfated/ total HS disaccharides (36.5\% in normal cartilage, 53\% in OA cartilage; Mann Whitney test, $P>0.05$ ) (Figure 6C).

Normal and OA cartilage did not differ significantly in the abundance of any single HS disaccharide (Figure 6D), or in the percentages of nonsulfated, monosulfated, disulfated, or triple-sulfated disaccharides (data not shown). However, the percentage of 6-O-sulfated disaccharides was increased in OA cartilage (19.8\% versus $12.1 \%$; Mann Whitney test, $P=0.0379$ ) (Figure $6 \mathrm{E}$ ). 
A
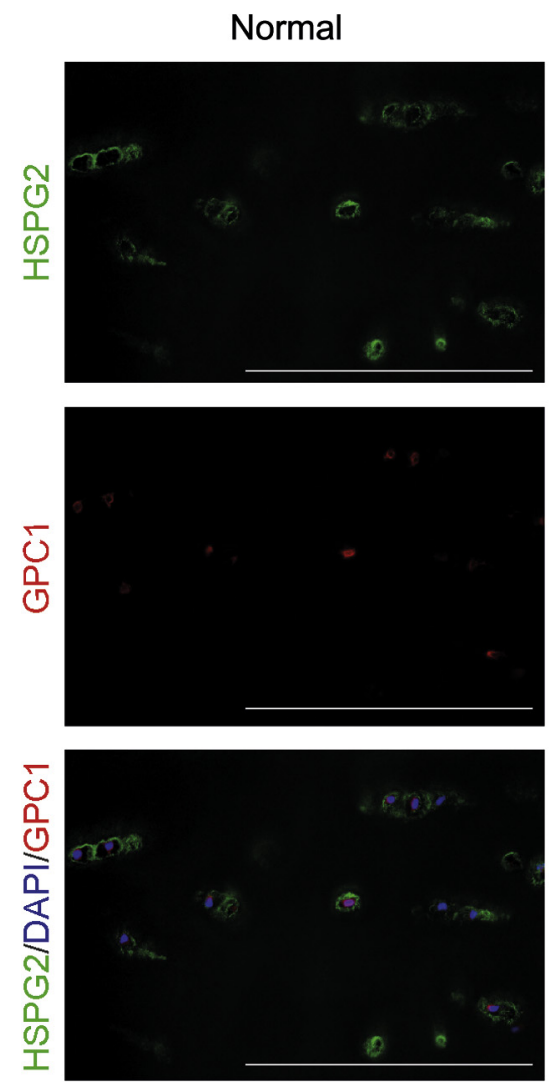

$O A$
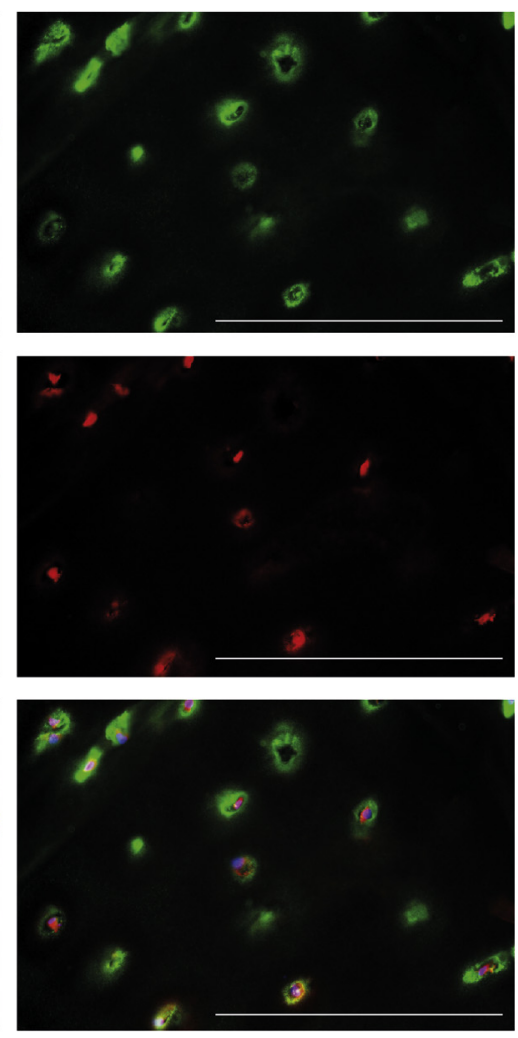

B
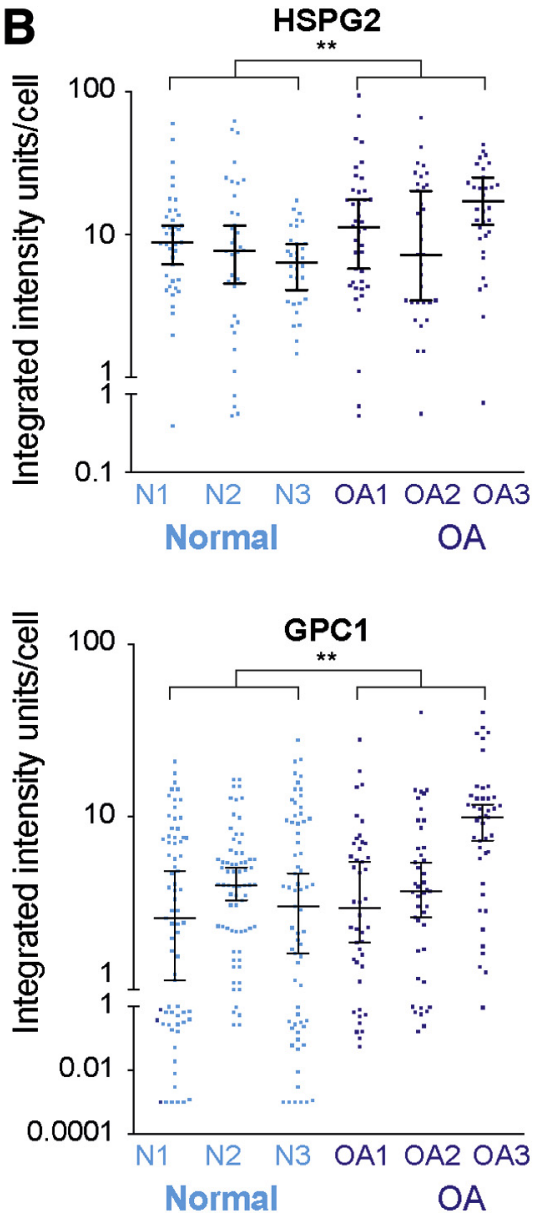

Figure 5 Expression of perlecan (heparan sulfate proteoglycan 2; HSPG2) and glypican 1 (GPC1) is increased in human osteoarthritic (0A) cartilage. A: Representative images showing expression of HSPG2 and GPC1 in human normal and OA cartilage. B: Expression of HSPG2 and GPC1 was semiquantitatively evaluated in three normal donors (N1, N2, and N3) and three 0 A donors (OA1, OA2, and $0 \mathrm{~A} 3)$, with each dot representing integrated fluorescence intensity per cell, calculated from at least six random fields of view on three sections per donor. For each donor, the median fluorescence intensity with $95 \%$ CIs of the mean are shown. ${ }^{* *} P<0.01$ (Kolmogorov-Smirnov test). Scale bars $=260 \mu \mathrm{m}(\mathbf{A})$.

\section{6-0-Sulfation of HS Correlates with Expression of GLCE, HS6ST1, EXTL2, and HS3ST3A1}

Correlation between expression of HS biosynthesis genes and HS disaccharide compositions were quantified.

The percentage of 6-O-sulfated disaccharides correlated with expression of HS6STl ( $r=0.74, P=0.0016)$ (Figure 6F), GLCE ( $r=0.775, P=0.0007)$ (Figure 6G), EXTL2 $(r=0.57, P=0.026)$, and HS3ST3Al $(r=0.575, P=0.025)$. The relationship between 6-Osulfation and HS6ST1 expression was linear, with a $4 \%$ increase in abundance of 6-O-sulfated disaccharides for every doubling in the amount of HS6ST1 (adjusted $\left.R^{2}=0.525, P=0.0014\right)$. This relationship was independent of age, OA, and sex. The relationship between 6O-sulfation and GLCE expression was linear, with a doubling in expression of $G L C E$ increasing the percentage of 6-O-sulfation by approximately $4 \%$. This relationship was dependent on age and sex, such that for every year increase in age there was a $0.17 \%$ increase in percentage 6-
O-sulfation, and that females had approximately $4 \%$ more 6-O-sulfation for the same amount of GLCE (adjusted $R^{2}=0.42, P=0.028$ ).

The percentage of 2-O-sulfated disaccharides correlated with expression of HS3ST3Al $(r=0.796, P=0.0004)$ and HS6ST1 $(r=0.57, P=0.026)$. The relationship between 2-O-sulfation and HS3ST3Al expression was linear, with a doubling in the levels of HS3ST3Al increasing the percentage of 2-O-sulfated disaccharides by $4 \%$. This association was OA and sex dependent, such that females with OA had a $0.62 \%$ increase in 2-O-sulfation for the same level of HS3ST3Al expression (adjusted $R^{2}=0.67, P=0.0036$ ).

Correlations were also examined between expression of HS biosynthesis genes and the relative abundance of individual disaccharide residues. This showed that HS3ST3Al expression correlated with the percentage of uronic acid (UA; 2-O-sulfate)-GlcNAc ( $r=0.772, P<0.05)$, UA(2O-sulfate $)-N$-sulfoglucosamine $(r=0.538, P<0.05)$, and $\mathrm{UA}-\mathrm{N}$-sulfoglucosamine(6-O-sulfate $) \quad(r=0.521$, 
A

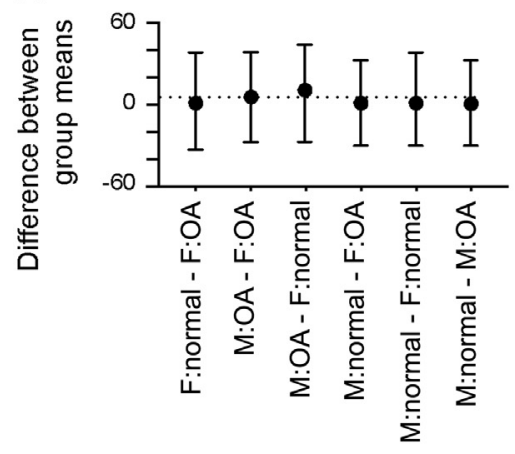

D

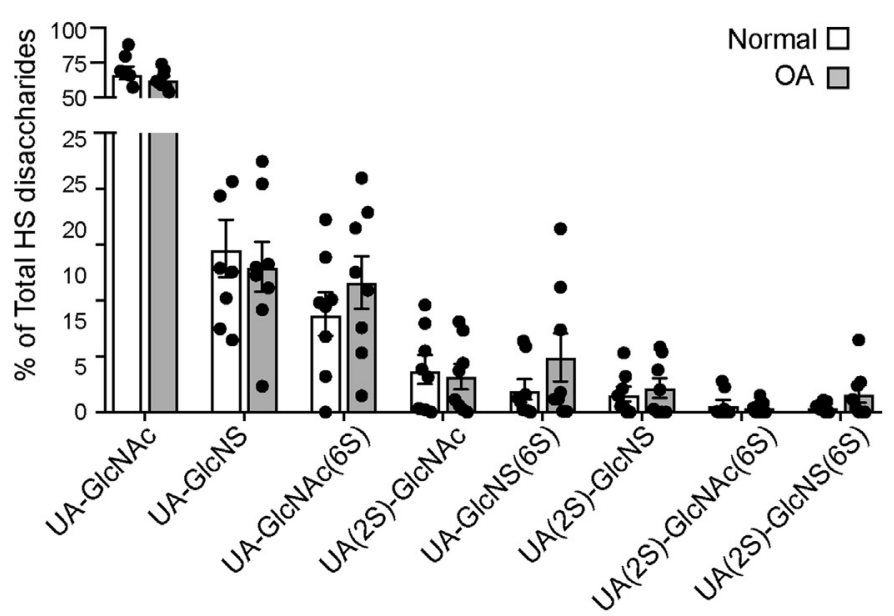

C

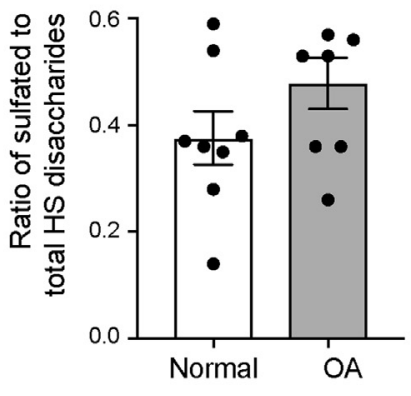

E

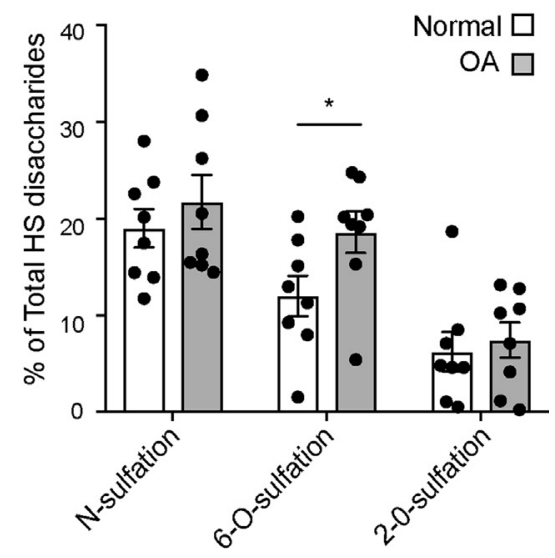

$\mathbf{F}$

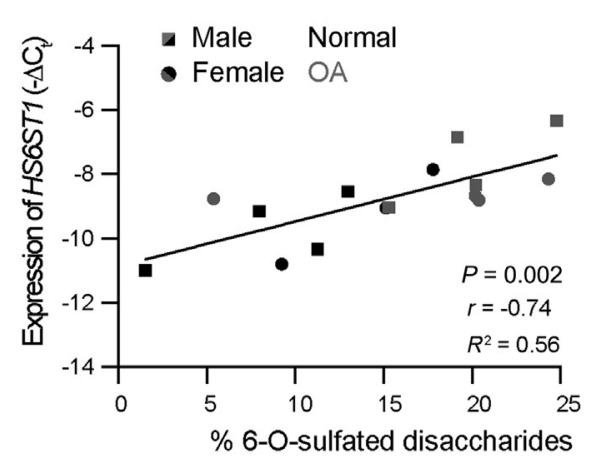

H

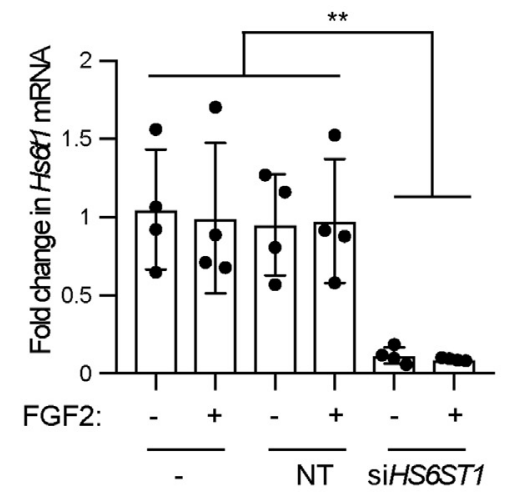

G

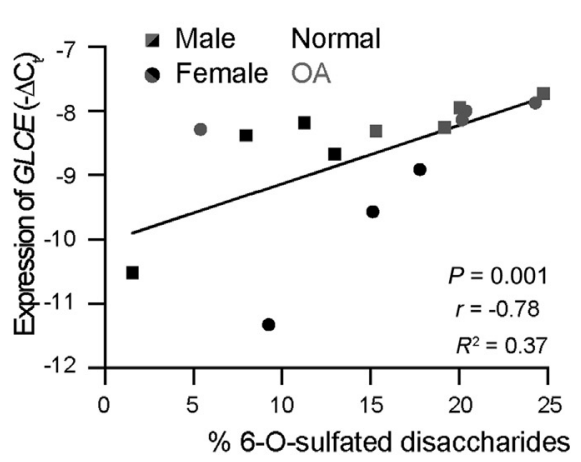


$P<0.05$ ), whereas HS6ST1 expression correlated with the percentage of UA(2-O-sulfate)-GlcNAc $(r=0.53$, $P<0.05)$, UA-GlcNAc(6-O-sulfate) $(r=0.58, P<0.05)$, and UA-GlcNAc $(r=-0.686, P=0.005)$.

Finally, HS3ST2 expression was found to be linearly associated with age, independent of $\mathrm{OA}$ and sex, with expression of $H S 3 S T 2$ increasing by $6 \%$ to $21 \%$ every year (adjusted $R^{2}=0.341, P=0.035$ ).

\section{siRNA Silencing of HS6ST1 Inhibits FGF2 Signaling}

To investigate whether changes in HS6STI expression and 6-O-sulfation have the capacity to alter signaling in OA chondrocytes, chondrocytes were isolated from knee cartilage of five donors with late-stage OA, and FGF2-dependent ERK phosphorylation was examined after siRNA knockdown of HS6ST1. siRNA treatment suppressed HS6STI mRNA levels by $>90 \%$ in all five donors (Figure $6 \mathrm{H}$ ). Addition of $20 \mathrm{ng} / \mathrm{mL}$ FGF2 stimulated rapid phosphorylation of ERK, and this was significantly reduced (by 40\%) in HS6STI-silenced cells (Figure 6I).

\section{Discussion}

HS proteoglycans control the retention, localization, and biological activity of $>400$ bioactive molecules, including growth factors (eg, FGF2 and TGF- $\beta$ ), morphogens (eg, BMP-4 and BMP-7), proteinases (eg, matrix metalloproteinase 13 and adamalysin with thrombospondin motifs 4 and 5), and proteinase inhibitors (eg, tissue inhibitor of metalloproteinases-3). ${ }^{4,35-37}$ Many HS ligands have significant impacts on cartilage homeostasis, making HS a critical regulator of joint health. Despite this important role, nothing was previously known about how the structure and sulfation pattern of HS may change during the development of osteoarthritis. This study shows that the expression of HS biosynthesis and modifying enzymes and the resulting HS disaccharide composition differs significantly in normal and osteoarthritic human knee cartilage.

Expression of the HS polymerases EXT1 and EXT2, as well as EXTL1 and EXTL2, was significantly increased in OA cartilage. The roles of these enzymes in homeostasis of adult cartilage have not been explored, but EXT1 and EXT2 are known to be important in musculoskeletal development. EXT1 regulates Indian hedgehog (Ihh) signaling during endochondral ossification, ${ }^{38}$ and mutations in both EXT1 and EXT2 are associated with development of hereditary multiple exostoses, an inherited pediatric disorder characterized by osteochondroma formation. ${ }^{39}$ Aberrant activation of chondrocyte hypertrophy has been implicated in development of OA, ${ }^{40}$ and may underlie the elevated expression of EXT1 and EXT2 observed. The role of the related EXTL genes in HS synthesis is less well understood, but they are thought to regulate early stages of HS chain elongation. ${ }^{41}$ Mutations in EXTL3 have been shown to impair musculoskeletal development, ${ }^{42}$ and although EXTL3 expression was not regulated in OA, expression of both EXTL1 and EXTL2 was strongly up-regulated.

Once polymerized, the HS chain can be modified by epimerization of GlcA to IdoA by a glucuronyl epimerase (GLCE) or by addition of sulfate groups to specific positions in the disaccharide units, by multiple sulfotransferases. The expression of 11 human HS sulfotransferases was measured, and it was found that the expression of NDST1, HS3ST1, and HS6ST1 was elevated in OA cartilage. Most notably, a significant increase was observed in the level of 6-Osulfated disaccharides in OA cartilage, which correlated strongly with expression of both HS6ST1 and GLCE. Epimerization of GlcA to IdoA by GLCE is thought to favor subsequent 6 -O-sulfation, ${ }^{43}$ suggesting that these two enzymes drive the increase in 6-O-sulfation in OA cartilage. Previous studies have shown that levels of 6-Osulfation are spatially and temporally regulated during development, ${ }^{18,19,21}$ and in diseases such as fibrosis, ${ }^{44,45}$ Alzheimer disease, ${ }^{22}$ and cancer. $^{46,47}$ In the case of fibrosis, a doubling in 6-O-sulfation is thought to exacerbate disease by amplifying TGF- $\beta^{44}$ and FGF2 ${ }^{45}$ signaling. We hypothesized that increased 6-O-sulfation in OA cartilage may similarly amplify downstream signaling pathways and alter tissue homeostasis. Consistent with this, using primary OA chondrocytes isolated from five different donors, it was found that siRNA silencing of HS6ST1 suppressed ERK phosphorylation in response to FGF2. This is in line with previous studies showing that 6-O-sulfation of HS promotes

\footnotetext{
Figure 6 6-0-sulfation increases in osteoarthritic (OA) cartilage. Heparan sulfate (HS) was isolated from normal and OA cartilage and its composition analyzed by disaccharide analysis. A: Forest plot showing there is no significant difference between the ages and sexes of normal and OA cartilage donors used for $\mathrm{HS}$ analysis. Bars show $95 \%$ CIs of the mean, dotted line shows $\mathrm{Y}=0$. B: The total amount of HS in OA cartilage is not statistically different from that in normal cartilage. C: The ratio of sulfated/total HS is not statistically different between the two groups of samples. D: Abundance of individual disaccharide species is not significantly different in normal and OA cartilage. E: 6-0-sulfated HS disaccharides are more abundant in OA cartilage, whereas levels of N- and 2-0-sulfation are not significantly altered. F: The level of 6-0-sulfation in donor cartilage correlates with expression of HS6ST1 (normal cartilage shown in black, OA cartilage in gray, males as squares, female samples as circles). G: The level of 6-0-sulfation in donor cartilage also correlates with expression of GLCE (normal cartilage shown in black, OA cartilage in gray, males as squares, female samples as circles). H: Expression of HS6ST1 in primary OA chondrocytes is silenced using siRNA. I: Silencing of HS6ST1 in primary OA chondrocytes reduces phosphorylation of extracellular signal-regulated kinase (p-ERK) in response to fibroblast growth factor 2 (FGF2; $20 \mathrm{ng} / \mathrm{mL}, 10$ minutes). $n=8(\mathbf{A}) ; n=15$ (F and $\mathbf{G}) ; n=5$ donors $(\mathbf{H})$. ${ }^{*} P<0.05, * * P<0.01$, and ${ }^{* * *} P<0.001$ (multiple $t$-tests, Benjamini, Krieger, and Yekutieli corrected). F, female; M, male; 2S, 2-0-sulfate; 6S, 6-0-sulfate; GlcNAc, N-acetyl glucosamine; GlcNS, $N$ sulfoglucosamine; NT, transfected with nontargeting siRNA; UA, uronic acid.
} 
FGF2 signaling, ${ }^{48-51}$ and confirms that changes in 6-Osulfation can alter biologically relevant signaling pathways in cartilage. 6-O-Sulfation is known to alter HS affinity for a range of ligands, ${ }^{52}$ with varying effects on their downstream activity. For example, 6-O-sulfation promotes TGF- $\beta^{53,54}$ signaling and inhibits $\mathrm{Wnt}^{55,56}$ and $\mathrm{BMP}^{57,58}$ signaling. An unbiased approach, such as phosphoproteomics, will be required to identify pathways regulated by $6-\mathrm{O}$-sulfation in normal and $\mathrm{OA}$ cartilage.

The importance of 6-O-sulfation is underlined by the fact that it is the only HS modification that is regulated at both the on and the off level, being added during HS biosynthesis in the Golgi apparatus by HS6ST1, HS6ST2, and/or HS6ST3 $3{ }^{43}$ and removed in the extracellular environment by the 6-O-endosulfatases SULF1 and/or SULF2 ${ }^{59}$ This permits remodeling of HS sulfation that is apparently critical to regulation of its functions. Indeed, increased expression of SULF1 and SULF2 was observed in OA cartilage, in line with previous reports examining

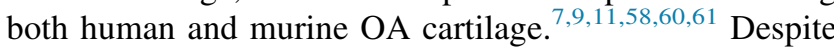
this, net levels of 6-O-sulfation were increased in our OA cartilage samples. As noted in recent studies from HS in muscle, ${ }^{27}$ this highlights the importance of analyzing HS structure directly and may indicate post-translational regulation of HS6ST and/or SULF activity, or differences in the catalytic efficiency, location, or stability of the enzymes. Sulf1 ${ }^{-1-}$ and Sulf $2^{-1-}$ mice developed accelerated $\mathrm{OA}$, and injection of recombinant SULF1 into the joint reduced cartilage damage, ${ }^{62}$ indicating the enzymes play a chondroprotective role. Elevated 6-O-sulfation may contribute to or perpetuate cartilage damage, potentially through driving anabolic pathways or impairing catabolic repair mechanisms.

No increase was seen in N-sulfation in OA cartilage, despite increased expression of NDST1 at the mRNA and protein level. The abundance of 3-O-sulfated disaccharides was not quantified because of their relative scarcity, but expression of $H S 3 S T l$ was elevated in OA samples. Further investigation may be warranted as knowledge of the functional impact of 3-O-sulfation and the extent of the 3-Oproteome expands. ${ }^{63,64}$

With regard to the HS core proteins, the previously reported increase in expression of HSPG $2,{ }^{6-9}$ glypican $1,{ }^{11}$ syndecan $1,{ }^{9-11}$ and syndecan $3^{65}$ was observed in OA cartilage, but not the previously observed increase in syndecan $4^{9,11-13}$ or decrease in agrin. ${ }^{66}$ This may suggest species- or joint-specific variation in expression of these genes. In addition, the expression of many HS-associated genes was significantly altered by isolation and culture of chondrocytes (data not shown), suggesting that HS biosynthesis is dynamically regulated by the chondrocyte microenvironment and that expression may change during the course of OA development.

A significant reduction was observed in the expression of TGFBR3 in OA cartilage. This HS core protein, alias betaglycan, does not have the ability to signal directly, but promotes TGF- $\beta$ signaling by recruiting the growth factor and presenting it to the type II TGF- $\beta$ receptor. In chondrocytes, TGFBR3 was recently shown to promote activation of latent TGF- $\beta$ in a connective tissue growth factorand HS-dependent manner. ${ }^{67}$ Reduction in expression of TGFBR3 in OA is thus likely to impair the chondroprotective effects of TGF- $\beta$ signaling.

Previous rodent microarray studies indicate that expression of some HS core proteins, biosynthesis, and modifying enzymes is regulated early in the course of OA development. $^{7,8,11,68-72}$ For example, expression of Extl ${ }^{11,68} \operatorname{Sdcl},{ }^{11,68,69} \mathrm{Gpcl},{ }^{1,69}$ and Hspg2 ${ }^{11,68}$ is significantly increased within 2 to 4 weeks of surgical OA induction (Supplemental Table S1) ${ }^{70-72}$ in line with our findings herein. Data on early changes in expression of sulfotransferases are variable, and early changes in Hs6st1 were not reported. This may suggest that expression of this gene is stimulated once cartilage damage starts to escalate but may also reflect differences between rodent post-traumatic $\mathrm{OA}$ and more variable human disease.

Age is a primary risk factor for the development of OA, and it is important to understand the cellular mechanisms that increase risk with age if we are to develop effective therapies to treat the disease. Previous studies have shown that HS sulfation patterns change with age in various tissues, including the aorta, ${ }^{73}$ myocardium, ${ }^{74}$ muscle stem cell niche, ${ }^{27}$ and brain, ${ }^{75,76}$ with concomitant changes in growth factor signaling. Although this study was not powered to detect changes in HS disaccharide composition with age, the expression of HS3ST2 in cartilage was found to increase with age, in contrast to what has previously been reported in skin. ${ }^{77}$ However, this observation is based on a low number of individuals $(n=11)$ because some values were missing for HS3ST2 expression. Further analysis of age-dependent changes in HS structure may identify changes that impair cartilage repair with age and so increase the risk of OA after injury.

Our analysis identified 6-O-sulfation as the primary HS motif that is altered in end-stage OA cartilage, validating it as a new target for potential therapeutic interventions. To understand the impact this change has on cartilage repair and OA progression, it will be necessary to perform unbiased analyses of the 6-O-sulfate-binding proteome in normal and OA cartilage. Comparative mass spectrometry approaches have proved useful in examining the 3-O-sulfate-binding proteome in cultured cells, ${ }^{64}$ and similar methods could be developed for cartilage analysis. Analysis of other joint tissues (eg, bone, synovium, meniscus) and earlier stages of OA progression can also be expected to shed further light on the role of HS in adult joint homeostasis.

\section{Supplemental Data}

Supplemental material for this article can be found at https://doi.org/10.1016/j.ajpath.2018.11.011. 


\section{References}

1. Sanchez-Adams J, Leddy HA, McNulty AL, O'Conor CJ, Guilak F: The mechanobiology of articular cartilage: bearing the burden of osteoarthritis. Curr Rheumatol Rep 2014, 16:451

2. Vincent TL, McLean CJ, Full LE, Peston D, Saklatvala J: FGF-2 is bound to perlecan in the pericellular matrix of articular cartilage, where it acts as a chondrocyte mechanotransducer. Osteoarthritis Cartilage 2007, 15:752-763

3. Vincent TL, Hermansson MA, Hansen UN, Amis AA, Saklatvala J: Basic fibroblast growth factor mediates transduction of mechanical signals when articular cartilage is loaded. Arthritis Rheum 2004, 50:526-533

4. Ori A, Wilkinson MC, Fernig DG: A systems biology approach for the investigation of the heparin/heparan sulfate interactome. J Biol Chem 2011, 286:19892-19904

5. Vincent TL: Targeting mechanotransduction pathways in osteoarthritis: a focus on the pericellular matrix. Curr Opin Pharmacol 2013, 13:449-454

6. Tesche F, Miosge N: Perlecan in late stages of osteoarthritis of the human knee joint. Osteoarthritis Cartilage 2004, 12:852-862

7. Karlsson C, Dehne T, Lindahl A, Brittberg M, Pruss A, Sittinger M, Ringe J: Genome-wide expression profiling reveals new candidate genes associated with osteoarthritis. Osteoarthritis Cartilage 2010, 18: 581-592

8. Sato T, Konomi K, Yamasaki S, Aratani S, Tsuchimochi K, Yokouchi M, Masuko-Hongo K, Yagishita N, Nakamura H, Komiya S, Beppu M, Aoki H, Nishioka K, Nakajima T: Comparative analysis of gene expression profiles in intact and damaged regions of human osteoarthritic cartilage. Arthritis Rheum 2006, 54:808-817

9. Loeser RF, Olex AL, McNulty MA, Carlson CS, Callahan MF, Ferguson CM, Chou J, Leng X, Fetrow JS: Microarray analysis reveals age-related differences in gene expression during the development of osteoarthritis in mice. Arthritis Rheum 2012, 64:705-717

10. Salminen-Mankonen H, Säämänen a-M, Jalkanen M, Vuorio E, Pirilä L: Syndecan-1 expression is upregulated in degenerating articular cartilage in a transgenic mouse model for osteoarthritis. Scand J Rheumatol 2005, 34:469-474

11. Gardiner MD, Vincent TL, Driscoll C, Burleigh A, Bou-Gharios G, Saklatvala J, Nagase H, Chanalaris A: Transcriptional analysis of micro-dissected articular cartilage in post-traumatic murine osteoarthritis. Osteoarthritis Cartilage 2015, 23:616-628

12. Barre P, Redinif F, Boumedienef K, Vielpeau C, Pujolf J: Semiquantitative reverse transcription-polymerase chain reaction analysis of syndecan- 1 and -4 messages in cartilage and cultured chondrocytes from osteoarthritic joints. Osteoarthritis Cartilage 2000, 8:34-43

13. Echtermeyer F, Bertrand J, Dreier R, Meinecke I, Neugebauer K, Fuerst M, Lee YJ, Song YW, Herzog C, Theilmeier G, Pap T: Syndecan-4 regulates ADAMTS-5 activation and cartilage breakdown in osteoarthritis. Nat Med 2009, 15:1072-1076

14. Guimond SE, Turnbull JE: Fibroblast growth factor receptor signalling is dictated by specific heparan sulfhate saccharides. Curr Biol 1999, 9:1343-1346

15. Shipp EL, Hsieh-Wilson LC: Profiling the sulfation specificities of glycosaminoglycan interactions with growth factors and chemotactic proteins using microarrays. Chem Biol 2007, 14:195-208

16. Ashikari-Hada S, Habuchi H, Kariya Y, Itoh N, Reddi AH, Kimata K: Characterization of growth factor-binding structures in heparin/heparan sulfate using an octasaccharide library. J Biol Chem 2004, 279:12346-12354

17. Kreuger J, Kjellén L: Heparan sulfate biosynthesis: regulation and variability. J Histochem Cytochem 2012, 60:898-907

18. Brickman Y, Nurcombe V, Gallagher J, Ford M, Turnbull J: Structural modification of FGF-binding HS at a determinative stage of neuroepithelial development. J Biol Chem 1998, 273:4350-4359

19. Kalus I, Rohn S, Puvirajesinghe TM, Guimond SE, EyckermanKölln PJ, Ten Dam G, van Kuppevelt TH, Turnbull JE, Dierks T:
Sulf1 and Sulf2 differentially modulate heparan sulfate proteoglycan sulfation during postnatal cerebellum development: evidence for neuroprotective and neurite outgrowth promoting functions. PLoS One 2015, 10:e0139853

20. Thompson SM, Fernig DG, Jesudason EC, Losty PD, van de Westerlo EM, van Kuppevelt TH, Turnbull JE: Heparan sulfate phage display antibodies identify distinct epitopes with complex binding characteristics: insights into protein binding specificities. J Biol Chem 2009, 284:35621-35631

21. Buresh-Stiemke RA, Malinowski RL, Keil KP, Vezina CM, Oosterhof A, Van Kuppevelt TH, Marker PC: Distinct expression patterns of Sulf1 and Hs6st1 spatially regulate heparan sulfate sulfation during prostate development. Dev Dyn 2012, 241: 2005-2013

22. Hosono-Fukao T, Ohtake-Niimi S, Hoshino H, Britschgi $\mathrm{M}$, Akatsu H, Hossain M, Nishitsuji K, van Kuppevelt T, Kimata K, Michikawa M, Wyss-Coray T, Uchimura K: Heparan sulfate subdomains that are degraded by Sulf accumulate in cerebral amyloid 8 plaques of Alzheimer's disease: evidence from mouse models and patients. Am J Pathol 2012, 180:2056-2067

23. Sepulveda-Diaz JE, Alavi Naini SM, Huynh MB, Ouidja MO, Yanicostas C, Chantepie S, Villares J, Lamari F, Jospin E, van Kuppevelt TH, Mensah-Nyagan AG, Raisman-Vozari R, SoussiYanicostas N, Papy-Garcia D: HS3ST2 expression is critical for the abnormal phosphorylation of tau in Alzheimer's disease-related tau pathology. Brain 2015, 138:1339-1354

24. Bernsen M, Smetsers T, van de Westerlo E, Ruiter D, Håkansson L, Gustafsson B, van Kuppevelt T, Krysander L, Rettrup B, Håkansson A: Heparan sulphate epitope-expression is associated with the inflammatory response in metastatic malignant melanoma. Cancer Immunol Immunother 2003, 52:780-783

25. Wijnhoven $\mathrm{T}$, Lensen $\mathrm{J}$, Rops $\mathrm{A}$, van der Vlag J, Kolset $\mathrm{S}$, Bangstad H, Pfeffer P, van den Hoven M, Berden J, van den Heuvel L, van Kuppevelt T: Aberrant heparan sulfate profile in the human diabetic kidney offers new clues for therapeutic glycomimetics. Am J Kidney Dis 2006, 48:250-261

26. Westergren-Thorsson G, Hedström U, Nybom A, Tykesson E, Åhrman E, Hornfelt M, Maccarana M, van Kuppevelt TH, Dellgren G, Wildt M, Zhou XH, Eriksson L, Bjermer L, Hallgren O: Increased deposition of glycosaminoglycans and altered structure of heparan sulfate in idiopathic pulmonary fibrosis. Int J Biochem Cell Biol 2017, 83:27-38

27. Ghadiali RS, Guimond SE, Turnbull JE, Pisconti A: Dynamic changes in heparan sulfate during muscle differentiation and ageing regulate myoblast cell fate and FGF2 signalling. Matrix Biol 2017, 59:54-68

28. Skidmore MA, Guimond SE, Dumax-Vorzet AF, Atrih A, Yates EA, Turnbull JE: High sensitivity separation and detection of heparan sulfate disaccharides. J Chromatogr A 2006, 1135:52-56

29. Barbáchano A, Ordóñez-Morán P, García JM, Sánchez A, Pereira F, Larriba MJ, Martínez N, Hernández J, Landolfi S, Bonilla F, Pálmer HG, Rojas JM, Muñoz A: SPROUTY-2 and E-cadherin regulate reciprocally and dictate colon cancer cell tumourigenicity. Oncogene 2010, 29:4800-4813

30. Arqués O, Chicote I, Tenbaum S, Puig I, Palmer HG: Standardized relative quantification of immunofluorescence tissue staining. Protocol Exchange 2012, doi:10.1038/protex.2012.008

31. Ismail HM, Miotla-Zarebska J, Troeberg L, Tang X, Stott B, Yamamoto K, Nagase H, Fosang AJ, Vincent TL, Saklatvala J: JNK2 controls aggrecan degradation in murine articular cartilage and the development of experimental osteoarthritis. Arthritis Rheum 2016, 68:1165-1171

32. Das KR, Imon AHMR: A brief review of tests for normality. Am J Theor Appl Stat 2016, 5:5-12

33. Benjamini Y, Krieger AM, Yekutieli D: Adaptive linear step-up procedures that control the false discovery rate. Biometrika 2006, 93:491-507 
34. Iozzo RV, Cohen IR, Grassel S, Murdoch AD: The biology of perlecan: the multifaceted heparan sulphate proteoglycan of basement membranes and pericellular matrices. Biochem J 1994, 302:625-639

35. Sakamoto S, Goldhaber P, Glimcher M: Mouse bone collagenase: the effect of heparin on the amount of enzyme released in tissue culture and on the activity of the enzyme. Calcif Tissue Res 1973, 12:247-258

36. Gao G, Plaas A, Thompson V, Jin S, Zuo F, Sandy J: ADAMTS4 (aggrecanase-1) activation on the cell surface involves C-terminal cleavage by glycosylphosphatidyl inositol-anchored membrane type 4-matrix metalloproteinase and binding of the activated proteinase to chondroitin sulfate and heparan sulfate on syndecan-1. J Biol Chem 2004, 279:10042-10051

37. Zeng W, Corcoran C, Collins-Racie L, Lavallie E, Morris E, Flannery C: Glycosaminoglycan-binding properties and aggrecanase activities of truncated ADAMTSs: comparative analyses with ADAMTS-5, -9, -16 and -18. Biochim Biophys Acta 2006, 1760:517-524

38. Koziel L, Kunath M, Kelly O, Vortkamp A: Ext1-dependent heparan sulfate regulates the range of Ihh signaling during endochondral ossification. Dev Cell 2004, 6:801-813

39. Pacifici M: The pathogenic roles of heparan sulfate deficiency in hereditary multiple exostoses. Matrix Biol 2018, 71-72:28-39

40. Sun MM, Beier F: Chondrocyte hypertrophy in skeletal development, growth, and disease. Birth Defects Res C Embryo Today 2014, 102: $74-82$

41. Busse-Wicher M, Wicher KB, Kusche-Gullberg M: The exostosin family: proteins with many functions. Matrix Biol 2014, 35:25-33

42. Notarangelo LD: Expanding the spectrum of skeletal dysplasia with immunodeficiency: a commentary on identification of biallelic EXTL3 mutations in a novel type of spondylo-epi-metaphyseal dysplasia. J Hum Genet 2017, 62:737-738

43. Habuchi H, Tanaka M, Habuchi O, Yoshida K, Suzuki H, Ban K, Kimata K: The occurrence of three isoforms of heparan sulfate 6-Osulfotransferase having different specificities for hexuronic acid adjacent to the targeted N-sulfoglucosamine. J Biol Chem 2000, 275:2859-2866

44. Lu J, Auduong L, White ES, Yue X: Up-regulation of heparan sulfate 6-O-sulfation in idiopathic pulmonary fibrosis. Am J Respir Cell Mol Biol 2014, 50:106-114

45. Alhasan AA, Spielhofer J, Kusche-Gullberg M, Kirby JA, Ali S: Role of 6-O-sulfated heparan sulfate in chronic renal fibrosis. J Biol Chem 2014, 289:20295-20306

46. Ferreras C, Rushton G, Cole CL, Babur M, Telfer BA, van Kuppevelt TH, Gardiner JM, Williams KJ, Jayson GC, Avizienyte E: Endothelial heparan sulfate 6-O-sulfation levels regulate angiogenic responses of endothelial cells to fibroblast growth factor 2 and vascular endothelial growth factor. J Biol Chem 2012, 287:36132-36146

47. Waaijer CJ, de Andrea CE, Hamilton A, van Oosterwijk JG, Stringer SE, Bovée JV: Cartilage tumour progression is characterized by an increased expression of heparan sulphate 60-sulphationmodifying enzymes. Virchows Arch 2012, 461:475-481

48. Pellegrini L, Burke DF, von Delft F, Mulloy B, Blundell TL: Crystal structure of fibroblast growth factor receptor ectodomain bound to ligand and heparin. Nature 2000, 407:1029-1034

49. Schlessinger J, Plotnikov AN, Ibrahimi OA, Eliseenkova AV, Yeh BK, Yayon A, Linhardt RJ, Mohammadi M: Crystal structure of a ternary FGF-FGFR-heparin complex reveals a dual role for heparin in FGFR binding and dimerization. Mol Cell 2000, 6:743-750

50. Pye D, Vives R, Turnbull J, Hyde P, Gallagher J: Heparan sulfate oligosaccharides require 6-O-sulfation for promotion of basic fibroblast growth factor mitogenic activity. J Biol Chem 1998, 273:22936-22942

51. Jemth P, Kreuger J, Kusche-Gullberg M, Sturiale L, GiménezGallego G, Lindahl U: Biosynthetic oligosaccharide libraries for identification of protein-binding heparan sulfate motifs: exploring the structural diversity by screening for fibroblast growth factor (FGF)1 and FGF2 binding. J Biol Chem 2002, 277:30567-30573

52. El Masri R, Seffouh A, Lortat-Jacob H, Vivès RR: The "in and out" of glucosamine 6-O-sulfation: the 6th sense of heparan sulfate. Glycoconj J 2017, 34:285-298
53. Lyon M, Rushton G, Gallagher J: The interaction of the transforming growth factor-betas with heparin/heparan sulfate is isoform-specific. J Biol Chem 1997, 272:18000-18006

54. Yue X, Li X, Nguyen HT, Chin DR, Sullivan DE, Lasky JA: Transforming growth factor-betal induces heparan sulfate 6-Oendosulfatase 1 expression in vitro and in vivo. J Biol Chem 2008, 283:20397-20407

55. Ai X, Do AT, Lozynska O, Kusche-Gullberg M, Lindahl U, Emerson CPJ: QSulf1 remodels the 6-O sulfation states of cell surface heparan sulfate proteoglycans to promote Wnt signaling. J Cell Biol 2003, 162:341-351

56. Dhoot G, Gustafsson M, Ai X, Sun W, Standiford D, Emerson CJ: Regulation of Wnt signaling and embryo patterning by an extracellular sulfatase. Science 2001, 293:1663-1666

57. Viviano BL, Paine-Saunders S, Gasiunas N, Gallagher J, Saunders S: Domain-specific modification of heparan sulfate by Qsulf1 modulates the binding of the bone morphogenetic protein antagonist Noggin. J Biol Chem 2004, 279:5604-5611

58. Otsuki S, Hanson SR, Miyaki S, Grogan SP, Kinoshita M, Asahara H, Wong C-H, Lotz MK: Extracellular sulfatases support cartilage homeostasis by regulating BMP and FGF signaling pathways. Proc Natl Acad Sci U S A 2010, 107:10202-10207

59. Morimoto-Tomita M, Uchimura K, Werb Z, Hemmerich S, Rosen S: Cloning and characterization of two extracellular heparin-degrading endosulfatases in mice and humans. J Biol Chem 2002, 277: 49175-49185

60. Otsuki S, Taniguchi N, Grogan SP, D'Lima D, Kinoshita M, Lotz M: Expression of novel extracellular sulfatases Sulf-1 and Sulf-2 in normal and osteoarthritic articular cartilage. Arthritis Res Ther 2008, 10:R61

61. Bateman JF, Rowley L, Belluoccio D, Chan B, Bell K, Fosang AJ, Little CB: Transcriptomics of wild-type mice and mice lacking ADAMTS-5 activity identifies genes involved in osteoarthritis initiation and cartilage destruction. Arthritis Rheum 2013, 65:1547-1560

62. Otsuki S, Murakami T, Okamoto Y, Hoshiyama Y, Oda S, Neo M: Suppression of cartilage degeneration by intra-articular injection of heparan sulfate 6-O endosulfatase in a mouse osteoarthritis model. Histol Histopathol 2017, 32:725-733

63. Huang Y, Mao Y, Zong C, Lin C, Boons GJ, Zaia J: Discovery of a heparan sulfate 3-O-sulfation specific peeling reaction. Anal Chem $2015,87: 592-600$

64. Thacker BE, Seamen E, Lawrence R, Parker MW, Xu Y, Liu J, Vander Kooi CW, Esko JD: Expanding the 3-O-sulfate proteome: enhanced binding of neuropilin-1 to 3-O-sulfated heparan sulfate modulates its activity. ACS Chem Biol 2016, 11:971-980

65. Pfander D, Swoboda B, Kirsch T: Expression of early and late differentiation markers (proliferating cell nuclear antigen, syndecan-3, annexin VI, and alkaline phosphatase) by human osteoarthritic chondrocytes. Am J Pathol 2001, 159:1777-1783

66. Eldridge S, Nalesso G, Ismail H, Vicente-Greco K, Kabouridis P, Ramachandran M, Niemeier A, Herz J, Pitzalis C, Perretti M, Dell'Accio F: Agrin mediates chondrocyte homeostasis and requires both LRP4 and $\alpha$-dystroglycan to enhance cartilage formation in vitro and in vivo. Ann Rheym Dis 2016, 75:1228-1235

67. Tang X, Muhammad H, McClean C, Zarebska J, Flemming J, Didangelos A, Önnerfjord P, Leask A, Saklatvala J, Vincent TL: Connective tissue growth factor (CTGF) contributes to joint homeostasis and osteoarthritis severity by controlling the matrix sequestration and activation of latent TGF $\beta$. Ann Rheum Dis 2018, 77:1372-1380

68. Loeser RF, Olex AL, McNulty MA, Carlson CS, Callahan M, Ferguson C, Fetrow JS: Disease progression and phasic changes in gene expression in a mouse model of osteoarthritis. PLoS One 2013, 8:e54633

69. Appleton CTG, Pitelka V, Henry J, Beier F: Global analyses of gene expression in early experimental osteoarthritis. Arthritis Rheum 2007, 56:1854-1868 
70. Geyer M, Grassel S, Straub RH, Schett G, Dinser R, Grifka J, Gay S, Neumann E, Muller-Ladner U: Differential transcriptome analysis of intraarticular lesional vs intact cartilage reveals new candidate genes in osteoarthritis pathophysiology. Osteoarthritis Cartilage 2009, 17: $328-335$

71. Dunn SL, Soul J, Anand S, Schwartz JM, Boot-Handford RP, Hardingham TE: Gene expression changes in damaged osteoarthritic cartilage identify a signature of non-chondrogenic and mechanical responses. Osteoarthritis Cartilage 2016, 24:1431-1440

72. Snelling S, Rout R, Davidson R, Clark I, Carr A, Hulley PA, Price AJ: A gene expression study of normal and damaged cartilage in anteromedial gonarthrosis, a phenotype of osteoarthritis. Osteoarthritis Cartilage 2014, 22:334-343

73. Feyzi E, Saldeen T, Larsson E, Lindahl U, Salmivirta M: Agedependent modulation of heparan sulfate structure and function. $\mathrm{J}$ Biol Chem 1998, 273:13395-13398

74. Huynh MB, Morin C, Carpentier G, Garcia-Filipe S, Talhas-Perret S, Barbier-Chassefière V, van Kuppevelt TH, Martelly I, Albanese P, PapyGarcia D: Age-related changes in rat myocardium involve altered capacities of glycosaminoglycans to potentiate growth factor functions and heparan sulfate-altered sulfation. J Biol Chem 2012, 287: $11363-11373$

75. Huynh MB, Villares J, Díaz JE, Christiaans S, Carpentier G, Ouidja MO, Sissoeff L, Raisman-Vozari R, Papy-Garcia D: Glycosaminoglycans from aged human hippocampus have altered capacities to regulate trophic factors activities but not $\mathrm{A} \beta 42$ peptide toxicity. Neurobiol Aging 2012, 33:1005.e11-1005.e22

76. Yamada T, Kerever A, Yoshimura Y, Suzuki Y, Nonaka R, Higashi K, Toida T, Mercier F, Arikawa-Hirasawa E: Heparan sulfate alterations in extracellular matrix structures and fibroblast growth factor-2 signaling impairment in the aged neurogenic niche. J Neurochem 2017, 142:534-544

77. Glass D, Viñuela A, Davies MN, Ramasamy A, Parts L, Knowles D, Brown AA, Hedman ÅK, Small KS, Buil A, Grundberg E, Nica AC, Meglio P, Nestle FO, Ryten M, Durbin R, McCarthy MI, Deloukas P, Dermitzakis ET, Weale ME, Bataille V, Spector TD: Gene expression changes with age in skin, adipose tissue, blood and brain. Genome Biol 2013, 14:R75 